\title{
Lifelong health insurance covers with surrender values: updating mechanisms in the presence of medical inflation
}

\author{
Jan Dhaene ${ }^{1}$, Els Godecharle ${ }^{1}$, Katrien Antonio ${ }^{1,2}$, Michel Denuit ${ }^{3}$, and Hamza Hanbali ${ }^{1}$ \\ ${ }^{1}$ Faculty of Economics and Business, KU Leuven, Belgium. \\ ${ }^{2}$ Faculty of Economics and Business, University of Amsterdam, The Netherlands. \\ ${ }^{3}$ Institut de Statistique, Biostatistique et Sciences Actuarielles, Université Catholique de \\ Louvain, Louvain-la-Neuve, Belgium.
}

March 31, 2017

\begin{abstract}
This paper considers the problem of a lifelong health insurance cover where medical inflation is not sufficiently incorporated in the level premium determined at policy issue. We focus on the setting where changes in health benefits, driven by medical inflation, are accounted for by an appropriate update or indexation of the level premium, the policy value, or both premium and policy value, during the term of the contract. Such an updating mechanism is necessary to restore the actuarial equivalence between future health benefits and surrender values on the one hand, and available policy values and future premiums on the other hand. We extend existing literature (Vercruysse et al. (2013) and Denuit et al. (2017)) by developing updating mechanisms in a discrete-time framework where medical inflation is only taken into account ex-post as it emerges over time and where surrender values are allowed for. We propose and design two types of surrender values: based on the ageing provision on the one hand and based directly on the premiums paid until surrender on the other hand. We illustrate our updating strategy with numerical examples, using Belgian data, and investigate the sensitivity of our findings with respect to elements from the technical basis (in particular: the lapse rates) used in the actuarial calculations. Our updating mechanism is generic and useful for a wide range of products in life and health insurance, where some elements of the technical basis are guaranteed while others are subject to revision according to policy conditions.
\end{abstract}

KEY WoRDS: medical expenses, lifelong health insurance contract, medical inflation index, lapse, surrender value, ageing provision. 


\section{Introduction}

This paper puts focus on lifelong health insurance contracts where periodic level premiums are paid. In such a contract the policyholder pays the same - hence: level - premium at the start of every policy period (e.g. yearly) in return for a lifelong cover against medical expenses. On the Belgian private health insurance market multiple examples of such contracts are present, see Devolder et al. (2008), which cover healthcare costs that are not refunded by the compulsory social security system in Belgium. According to Assuralia (2016), the professional association of insurance companies in Belgium, over 5.5 million Belgian citizens entered a private health insurance contract in 2014. Also the German market features such contracts financed with a whole-life level premium that is contractually fixed until the policyholder dies, lapses or changes her cover (Christiansen et al. (2016)).

At contract inception, the level premium is calculated so that the contract is actuarially fair, i.e. the actuarial value (or expected present value) of premiums over the contract duration is equal to the actuarial value of the health benefits and the possible surrender value paid out to the insured in case of policy cancellation. As medical expenses typically rise over the lifetime, a level premium contract generates premium surpluses in the early years which lead to asset accumulation in a policy value (also called: reserve or ageing provision) ${ }^{1}$, while the shortfall of premiums in later years is covered by these assets. As a result, the well-known hump-shape of the policy values becomes apparent, see Dickson et al. (2013) (Chapter 7) for a discussion of policy values with a focus on life insurance products. Christiansen et al. (2016) denote this health insurance funding strategy as individual whole-life balancing and stress that this is fundamentally different from guaranteed renewable short-term health insurance (Pauly et al. (1995)) as well as time-consistent health insurance (Cochrane (1995)).

Unpredictable changes in prices for medical goods and services impact the health benefits that will be paid over the course of a lifelong health insurance policy. Given the long-term nature of health insurance contracts and the impossibility to predict or hedge against medical inflation, insurers are generally not able to precisely account for this medical inflation in the calculation of the level premium at policy issue. Therefore these lifelong contracts are usually designed in such a way that the insurer is allowed to adapt or index the premium at regular times (e.g. yearly) to account for medical inflation not taken into account at policy issue, based on some predefined medical inflation index. This practice is used in several EU member countries, for instance in Belgium $^{2}$ and Germany (Milbrodt (2005), Devolder et al. (2008) and Pitacco (2014)).

Vercruysse et al. (2013) and Denuit et al. (2017) consider the problem of premium indexing in the presence of medical inflation for lifelong health insurance contracts with non-transferable reserves. Non-transferability of the reserves means that an insured who is lapsing the contract or wants to switch to another insurance company is not allowed to take any fraction of the reserve (or: ageing provision) with her, neither is any other surrender value paid. This is the current situation on e.g. the Belgian market of lifelong health covers. The absence of a

\footnotetext{
${ }^{1}$ We use the term reserve in this paper, but policy value, and ageing provision are synonyms often used in the literature.

${ }^{2}$ The Royal Decree of March 182016 stipulates that the medical inflation index for the Belgian health insurance market is published yearly, see http://statbel.fgov.be/nl/statistieken/cijfers/economie/ gezondheidssector/. In Belgium, individual private health coverages are lifelong by law and, in case of level premiums, the initial premium amount is fixed at policy issue. Health insurers may update their premiums yearly, at the premium due date, on the basis of the evolution of the aggregate consumer price index (CPI) or the specific medical index.
} 
surrender value has a premium-reducing effect in case the insurer accounts for lapses in the premium calculations. This is often considered as controversial since lapse rates are difficult to estimate and predict, and may have a severe impact on the insurer's financial situation (Dickson et al. (2013), Christiansen et al. (2016)). Another drawback of the absence of a surrender value is that the insured is tied to her insurer, especially at times when accumulated assets in the form of a policy value are relatively high. Hofmann \& Browne (2013) provide empirical evidence of the lock-in consumers face when premiums are front-loaded.

Although non-transferability of reserves can be actuarially fair when appropriately taken into account in the premium calculation, and is in line with e.g. current Belgian regulation, consumers may feel this lack of liquidity of their contract as a serious drawback. Hence it is worth to investigate actuarial models where transferability of (part of) the ageing provision is allowed in case of surrender. Baumann et al. (2008) study how transferable ageing provisions can be designed without imposing premium charges on the policyholders staying in the contract. Obviously, an insurance market allowing for transferability of ageing provisions on health insurance contracts would require an appropriate set of regulations to frame such transfers.

Our paper studies the setting of a lifelong health insurance cover which allows for a surrender value. As a first contribution, we propose two possible definitions for this surrender value. The first definition is based on the ageing provision built up by the policyholder until the moment of surrender. The second definition directly takes the premiums paid up to surrender into account. As a second contribution, we consider the problem of medical inflation in a context of private health insurance contracts with surrender values and propose updating mechanisms which generalize the results of Vercruysse et al. (2013) and Denuit et al. (2017). In this setting, several ways exist to restore the actuarial equivalence in the presence of unanticipated medical inflation. The insurer can either update premiums, update the reserve, or combine both approaches. In the first case it is the insured who carries the burden of increased costs due to medical inflation, in the second case it is the insurer, while in the third case they share the burden. We provide explicit solutions for the initial premium and the updating strategy with the specific surrender values designed in this paper. This general approach is demonstrated in numerical examples, based on Belgian data.

Setting up an appropriate model for lapse rates is a difficult task: data may be scarce, various risk factors can influence lapse rates and the impact of lapse rates on the financial situation of the insurer can be high. For example, Kuo et al. (2003) explore the impact of unemployment and interest rate on lapse rates. Hofmann \& Browne (2013) show that policyholders generally lapse less in case of higher premium front-loading and Christiansen et al. (2016) find that premium development, premium adjustment frequency and the sales channel impact lapse rates. In the present work, we investigate how the choice of the lapse rates affects our numerical results by means of a sensitivity analysis. This is the third contribution of our paper.

The remainder of this paper is organized as follows. In Section 2, we describe the lifelong health insurance contract under study. In Section 3, we extend the framework of Vercruysse et al. (2013) to take into account surrender values. We describe how contracts may be updated over time to take unanticipated medical inflation into account. In Section 4 , we consider the special cases of reserve- and premium-dependent surrender values, respectively. Section 5 discusses detailed numerical examples. Section 6 concludes the paper. 


\section{The lifelong health insurance contract}

\subsection{Health benefits and surrender values}

The policyholder's (integer) age at policy issue (i.e. time 0 ) is denoted by $x$, so that upon survival at time $k$, she has reached age $x+k$. We denote the ultimate integer age by $\omega$, assumed to be finite. Survival until integer age $\omega$ then has a positive probability, whereas survival until integer age $\omega+1$ has probability zero.

The superscript "(0)" denotes quantities estimated or known at policy issue. Similar to the setting of Baumann et al. (2008) and Christiansen et al. (2016) we use age-specific estimates of healthcare expenses to price the contract. The average healthcare benefit for a policyholder aged $x+j$ in the year $(j, j+1), j \in\{0,1, \ldots, \omega-x\}$, is denoted by $b_{x+j}^{(0)}$. The quantity $b_{x+j}^{(0)}$ is also called risk premium or expected claim amount per policy. Of course, some policyholders will require a higher payout, whereas other policyholders will not produce any claim. The approach adopted here consists in averaging out the claims over all policyholders with similar characteristics; see e.g. Pitacco (2014) for more details on the statistical estimation of $b_{x+j}^{(0)}$. Furthermore, in case the policyholder cancels the contract in year $(j, j+1)$, the surrender value $w_{x+j+1}^{(0)}$ is paid out at the end of the year. We set $w_{\omega+1}^{(0)}=0$, which means that the surrender value in the last possible year of survival is equal to zero. In Section 4.1 and 4.2 we design two meaningful surrender values for this type of contract.

The healthcare expenses covered by the contract are subject to medical inflation. Medical inflation, as we define it, accounts for the full increase of medical costs, not only the increase of these medical costs above the inflation taken into account by the aggregate consumer price index. We assume that medical inflation is unpredictable and hence, at policy issue, an assumption has to be made about this inflation. In our setting the actuary includes a constant and ageindependent future medical inflation of $f$ per year in the premium calculation. This implies

$$
b_{x+j}^{(0)}=(1+f)^{j} \cdot \bar{b}_{x+j}^{(0)},
$$

where $\bar{b}_{x+j}^{(0)}$ is the estimate at time 0 of the average medical expenses in year $(0,1)$ for a person aged $x+j$ at policy issue. We assume that appropriate estimates for the values $\bar{b}_{x+j}^{(0)}$ are available at time 0 . Hence, $(1+f)^{j} \cdot \bar{b}_{x+j}^{(0)}$ is the estimate at time 0 of the expected medical expenses in year $(j, j+1)$ for an $(x+j)$ year old in the beginning of that year. Both the surrender value $w_{x+j+1}^{(0)}$ and the level premium $\pi^{(0)}$ determined at policy issue incorporate a medical inflation of $f$ per year. In the context of temporary health covers, $f$ is sometimes set equal to 0 even though this may be a naive assumption, depending on the market circumstances. Due to the long term nature of the contracts studied in this paper, we consider it appropriate to work with a more realistic estimate $f>0$.

Obviously, observed medical inflation may and will likely depart from the assumed $f$. Our contract assumptions stipulate that premiums, reserves and possibly also surrender values may be updated (on a yearly basis) according to a well-defined procedure, in order to restore the actuarial equivalence that is broken due to unanticipated medical inflation. We use the superscript " $(k)$ " to denote the updated values based on actual inflation observed up to and including time $k$, where future inflation is assumed to be $f$ per year. The updated value at time $k$ for the 


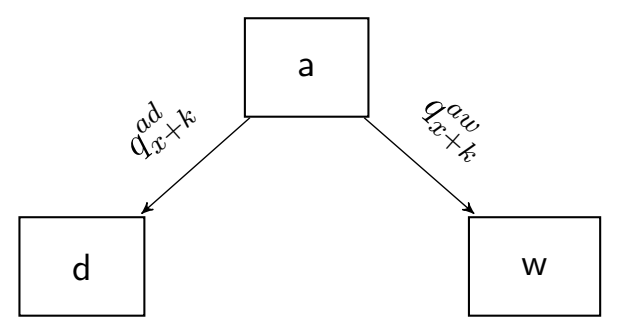

Figure 1: The two-decrement model describing the lifelong health insurance policy.

health benefit estimates in the year $(k+j, k+j+1)$ is denoted by $b_{x+k+j}^{(k)}, k \in\{0,1, \ldots, \omega-x\}$ and $j=0,1, \ldots, \omega-x-k$. Hence,

$$
b_{x+k+j}^{(k)}=(1+f)^{j} \cdot \bar{b}_{x+k+j}^{(k)},
$$

where $\bar{b}_{x+k+j}^{(k)}$ is the average health expenditure for an insured aged $(x+k+j)$ in year $(k, k+1)$. Appropriate estimates for the values $\bar{b}_{x+k+j}^{(k)}$ are available at time $k$. Consequently, $b_{x+k+j}^{(k)}$ is our estimate at time $k$ for the expected medical expenses in year $(k+j, k+j+1)$ for a policyholder aged $(x+k+j)$ in the beginning of that year. Furthermore, $w_{x+k+j+1}^{(k)}$ is the time $k$ updated value of the surrender value that could be paid at time $k+j+1$, taking into account the observed inflation until time $k$ and an assumed inflation of $f$ per year beyond that time. $w_{\omega+1}^{(k)}=0$ in line with the assumption of ultimate age $\omega$. The corresponding updated premium is $\pi^{(k)}$ to be paid from time $k$ on.

Apart from the assumed medical inflation, other elements of the technical basis (interest, mortality and lapse rates) are assumed to be in line with the reality that unfolds over time. As such, these elements do not require a yearly update in order to maintain actuarial equivalence. This assumption allows us to isolate and investigate the effect of medical inflation on its own. As a final comment, we stress that we do not revise the assumed inflation $f$ during the coverage period. This revision is achieved ex-post by the proposed indexing mechanism.

\subsection{Discrete-time double decrement model}

We describe the lifelong health insurance policy introduced in Section 2.1 as a two-decrement Markov model, with states "active" (i.e. policy in force), "withdrawn" (i.e. policy has been cancelled) and "dead", abbreviated as "a", "w" and "d", respectively. Figure 1 gives a graphical representation. $X_{k}$ denotes the status of the contract at time $k$, starting from $X_{0}=a$. The stochastic process $\left\{X_{k}, \quad k=0,1,2, \ldots\right\}$ describes the states occupied over time.

For $j$ and $k \in\{0,1,2, \ldots\}$, we define the sojourn (or non-exit) probability ${ }_{j} p_{x+k}^{a a}$ as

$$
{ }_{j} p_{x+k}^{a a}=\operatorname{Pr}\left[X_{k+j}=a \mid X_{k}=a\right] .
$$

In words, (2) is the probability that a policy in force at age $x+k$ is still in force $j$ years later. The probability that a policy in force at age $x+k$ has ceased $j$ years later (due to death or surrender), is denoted by ${ }_{j} q_{x+k}^{a a}$. This "exit" probability can be expressed as

$$
{ }_{j} q_{x+k}^{a a}=\operatorname{Pr}\left[X_{k+j} \neq a \mid X_{k}=a\right]=1-{ }_{j} p_{x+k}^{a a} .
$$


We also introduce the probabilities ${ }_{j} q_{x+k}^{a d}$ and ${ }_{j} q_{x+k}^{a w}$, defined as

$$
{ }_{j} q_{x+k}^{a d}=\operatorname{Pr}\left[X_{k+j}=d \mid X_{k}=a\right] \text { and }{ }_{j} q_{x+k}^{a w}=\operatorname{Pr}\left[X_{k+j}=w \mid X_{k}=a\right] .
$$

These are the probabilities of leaving the portfolio due to respectively death and surrender between ages $x+k$ and $x+k+j$.

The following relations are well known

$$
{ }_{j} p_{x+k}^{a a}+{ }_{j} q_{x+k}^{a d}+{ }_{j} q_{x+k}^{a w}=1
$$

and

$$
{ }_{j+1} p_{x+k}^{a a}=\prod_{l=0}^{j} p_{x+k+l}^{a a} .
$$

In accordance with standard actuarial notation, we omit the left subindex when it is equal to one, e.g. ${ }_{1} p_{x+k}^{a a}=p_{x+k}^{a a}$. The ultimate integer age $\omega$ is such that $p_{\omega-1}^{a a}>0$, while $p_{\omega}^{a a}=0$.

\subsection{Actuarial value of premiums, health benefits and surrender values, and the equivalence principle}

Although the actuarial literature (Haberman \& Pitacco (1999), Dickson et al. (2013)) presents many possible multi-state models to design a health insurance product, we follow current practice in the Belgian and German market of lifelong health insurance covers (Devolder et al. (2008), Milbrodt (2005)) and use the two-decrement model introduced in Section 2.2. Within this setting premiums are paid as long as the policy is in force and an estimate of healthcare expenses over the insured's lifetime is used to value the benefits. The valuation of the benefits hence assumes an approximate, average and age-specific health benefit that is guaranteed as long as the policy is active. We do not take expenses into account, but it is straightforward to extend our work as such. We assume a constant yearly technical interest rate $i$, and denote the corresponding annual discount factor $(1+i)^{-1}$ by $v$. With premiums being paid at the beginning of the year, let

$$
\ddot{a}_{x}^{a a}=\sum_{l=0}^{\omega-x} \iota p_{x}^{a a} v^{l}
$$

be the actuarial value at policy issue of an annuity-due paying an amount of 1 per year to an $(x)$ year old as long as the health insurance contract is in force. Furthermore, let

$$
B_{x}^{(0)}=\sum_{j=0}^{\omega-x}{ }_{j} p_{x}^{a a} v^{j} b_{x+j}^{(0)}
$$

be the actuarial value at time 0 of all health-related benefits for a single policy. As discussed in Section $2.1, b_{x+j}^{(0)}$ is an estimate (set at time 0 ) of the average medical expenses in year $(j, j+1)$ for an $(x+j)$ year old in the beginning of that year. In Section 5 we present a typical agespecific evolution of these average costs, inspired by Belgian market data. Healthcare benefits are paid at the beginning of the year in our pricing model, which is a convenient and conservative assumption. Let

$$
W_{x}^{(0)}=\sum_{j=0}^{\omega-x}{ }_{j} p_{x}^{a a} q_{x+j}^{a w} v^{j+1} w_{x+j+1}^{(0)}
$$


be the actuarial value at time 0 of the surrender option.

At policy issue the level premium $\pi^{(0)}$ for a policyholder aged $x$ is determined from the actuarial equivalence principle

$$
\pi^{(0)} \ddot{a}_{x}^{a a}=B_{x}^{(0)}+W_{x}^{(0)},
$$

taking into account the information available at policy issue (i.e. at time 0), including a deterministic medical inflation of $f$ per year.

Solving equation (10) for $\pi^{(0)}$ leads to the level premium to be paid yearly in advance. However, the equivalence relation does not always provide an explicit expression for this premium. This is the case for instance when the surrender value, and hence also $W_{x}^{(0)}$, is defined in terms of the available reserve or in terms of the premiums paid so far. In Sections 4.1 and 4.2 , we design such surrender values and present a methodology which gives an explicit expression for $\pi^{(0)}$.

\section{Updating the health insurance contract}

This section explains the generic updating mechanism for the lifelong health insurance contract as described in Section 2. We first generalize the notation introduced in Section 2.3. Recall that the contract was underwritten at time 0 by an insured aged $x$ at that time. For $k \in$ $\{0,1,2, \ldots, \omega-x\}$ and $j \in\{0,1,2, \ldots, \omega-x-k\}$, let

$$
B_{x+k+j}^{(k)}=\sum_{l=0}^{\omega-x-k-j} l p_{x+k+j}^{a a} v^{l} b_{x+k+j+l}^{(k)}
$$

be the actuarial value at time $k+j$ of the health benefits to be paid at time $k+j$ and beyond for a policy still in force at time $k+j$. Similarly, let

$$
W_{x+k+j}^{(k)}=\sum_{l=0}^{\omega-x-k-j} \imath_{x+k+j}^{a a} q_{x+k+j+l}^{a w} v^{l+1} w_{x+k+j+l+1}^{(k)}
$$

be the actuarial value at time $k+j$ of the surrender option for a policy still in force at time $k+j$. As outlined in Section 2, (11) and (12) use the superscript "(k)" and hence take into account the observed medical inflation until time $k$, while a constant yearly medical inflation of $f$ is assumed from time $k$ on.

\subsection{Updating mechanism: available and required reserve}

The proposed updating mechanism is based on recursive formulas for the reserve that are well known in the literature on life insurance mathematics, see Dickson et al. (2013) (Chapter 7). We extend these formulas to lifelong health insurance contracts with surrender values and develop an updating mechanism which brings the available reserve $(V)$ to the level of the required reserve

$(\vec{V})$. The available reserve should be understood as the available assets or the retrospective reserve, whereas the required reserve represents the actuarial liabilities taking into account future premiums, i.e. the prospective reserve.

On the one hand, the relation

$$
V_{x+k}^{(k-1)}=\left(V_{x+k-1}^{(k-1)}+\pi^{(k-1)}-b_{x+k-1}^{(k-1)}-q_{x+k-1}^{a w} v w_{x+k}^{(k-1)}\right)\left(v p_{x+k-1}^{a a}\right)^{-1},
$$


allows us to determine the available reserve at time $k$ (before updating) from the available reserve $V_{x+k-1}^{(k-1)}$ at time $k-1$ and cash in- and outflows in the past year. On the other hand, we express the required reserve at time $k$ as the expected present value of the benefits minus the expected present value of the premiums

$$
\vec{V}_{x+k}^{(k)}=B_{x+k}^{(k)}+W_{x+k}^{(k)}-\pi^{(k)} \ddot{a}_{x+k}^{a a} .
$$

If the actuarial assumptions in the technical basis are fulfilled over time, (13) and (14) coincide. When reality departs from the assumptions made in the technical basis, (13) and (14) may differ. This breaks the actuarial equivalence and updating mechanisms are necessary to restore the equivalence.

\subsection{Updating mechanism: from time $k-1$ to time $k$}

Suppose that the policy is still in force at time $k \in\{1,2,3, \ldots, \omega-x\}$. Reevaluations up to time $k-1$ have led to the updated values $b_{x+k+j}^{(k-1)}$ and $w_{x+k+j+1}^{(k-1)}, j \in\{0,1,2, \ldots, \omega-x-k\}$, for the health-related benefits and the surrender values, respectively. Here, $b_{x+k+j}^{(k-1)}$ and $w_{x+k+j+1}^{(k-1)}$ are based on the observed medical inflation until time $k-1$, while assuming a medical inflation of $f$ per year from time $k-1$ on. Furthermore, premiums have been adapted from year to year and have reached level $\pi^{(k-1)}$ at time $k-1$.

At time points $1, \ldots, k-1$ updates were made to rebalance the available and the required reserve. In particular, at time $k-1$ we restored the actuarial equivalence such that

$$
V_{x+k-1}^{(k-1)}=\vec{V}_{x+k-1}^{(k-1)}
$$

where

$$
\vec{V}_{x+k-1}^{(k-1)}=B_{x+k-1}^{(k-1)}+W_{x+k-1}^{(k-1)}-\pi^{(k-1)} \ddot{a}_{x+k-1}^{a a},
$$

with $B_{x+k-1}^{(k-1)}$ and $W_{x+k-1}^{(k-1)}$ defined according to (11) and (12), respectively. The right-hand side in (16) is the actuarial value at time $k-1$ of the future liabilities of the contract, based on the information available at that time. Splitting the payments related to year $(k-1, k)$ from the other payments, we obtain the recursive equation for the required reserve

$$
\begin{aligned}
\vec{V}_{x+k-1}^{(k-1)}= & b_{x+k-1}^{(k-1)}+q_{x+k-1}^{a w} v w_{x+k}^{(k-1)}-\pi^{(k-1)} \\
& +p_{x+k-1}^{a a} v\left(B_{x+k}^{(k-1)}+W_{x+k}^{(k-1)}-\pi^{(k-1)} \ddot{a}_{x+k}^{a a}\right) .
\end{aligned}
$$

Similar recursive expressions are well known in life insurance mathematics, see Dickson et al. (2013). Having arrived at time $k$, the available reserve for a policy still in force at age $x+k$, taking into account all information up to time $k-1, V_{x+k}^{(k-1)}$, is given by (13). Combining this equation with (15) and (17) we find the following expression for the available reserve $V_{x+k}^{(k-1)}$

$$
V_{x+k}^{(k-1)}=B_{x+k}^{(k-1)}+W_{x+k}^{(k-1)}-\pi^{(k-1)} \ddot{a}_{x+k}^{a a} .
$$

Thus, the available reserve (left hand side) and the required reserve (right hand side) at time $k$ are equal, provided the technical basis that was used at time $k-1$ is still adapted at time $k$. This result is known to hold in general, see e.g. Dickson et al. (2013). 
Suppose now that medical inflation during year $(k-1, k)$ was such that each future health benefit $b_{x+k+j}^{(k-1)}, j \in\{0,1, \ldots, \omega-x-k\}$, has to be replaced by the corresponding adapted health benefit $b_{x+k+j}^{(k)}$, determined at time $k$. The latter assumes a future yearly medical inflation $f$, while taking into account observed medical inflation up to time $k$. In particular, due to medical inflation, the actuarial values of future health benefits $B_{x+k}^{(k-1)}$ and surrender benefits $W_{x+k}^{(k-1)}$ have to be replaced by $B_{x+k}^{(k)}$ and $W_{x+k}^{(k)}$, respectively, which are based on observed medical inflation until time $k$, see (11). Due to this change in the health benefits, the actuarial equivalence is broken at time $k$, in the sense that the available reserve is different from the actuarial value of future liabilities (i.e. the required reserve) at that time, i.e.

$$
V_{x+k}^{(k-1)} \neq B_{x+k}^{(k)}+W_{x+k}^{(k)}-\pi^{(k)} \ddot{a}_{x+k}^{a a} .
$$

\subsection{Restoring the actuarial equivalence: premium updates, reserve updates or shared burden}

We investigate how the broken actuarial equivalence in (19) can be restored at time $k$ through an update of the premium level or the available reserve. From time $k$ on, the level premium $\pi^{(k-1)}$ that was determined at time $k-1$, is replaced by the updated level premium $\pi^{(k)}$. These premium updates $\pi^{(k)}-\pi^{(k-1)}$ are financed by the policyholder. Also, at time $k$, the available reserve $V_{x+k}^{(k-1)}$ is updated to $V_{x+k}^{(k)}$. Obviously, this reserve update is financed by the insurer. The reserve update depends on the updated premium level and vice versa through (13). Restoring the actuarial equivalence may be obtained in many ways, in the sense that an infinite number of pairs $\left(V_{x+k}^{(k)}, \pi^{(k)}\right)$ satisfy the relation

$$
V_{x+k}^{(k)}=B_{x+k}^{(k)}+W_{x+k}^{(k)}-\pi^{(k)} \ddot{a}_{x+k}^{a a} .
$$

When $\pi^{(k)}=\pi^{(k-1)}$ the insurer carries all the effects of medical inflation, whereas $V_{x+k}^{(k)}=V_{x+k}^{(k-1)}$ lays all the uncertainty with the policyholder. Both parties can also share the burden. A corresponding increase $W_{x+k}^{(k)}-W_{x+k}^{(k-1)}$ is financed by the insurer (via a reserve increase) and/or the policyholder (via increased premiums).

Let us briefly discuss two extreme cases where the effect of inflation is entirely borne by one of the agents, either the insurer or the policyholder.

Example 1. When the premium is kept unchanged, i.e. when $\pi^{(k)}=\pi^{(k-1)}$, we find from (18) and (20) that

$$
V_{x+k}^{(k)}-V_{x+k}^{(k-1)}=\left(B_{x+k}^{(k)}-B_{x+k}^{(k-1)}\right)+\left(W_{x+k}^{(k)}-W_{x+k}^{(k-1)}\right),
$$

which means that the health benefit and surrender value increases are completely financed by the insurer via an increase of the available assets. In practice, a reserve increase may be financed by technical gains on interest, mortality or surrenders. For example, technical gains arise in case realized interest rates are higher than the ones assumed in the technical basis. Mortality gains may originate when actual mortality is higher than mortality assumed in the technical basis or increasing longevity postpones late-life costs to higher ages. Although not considered in this paper, technical gains can also arise when actual expenses are lower than the expenses assumed for the premium calculation. 
Example 2. When the insurer does not increase the available reserve, i.e. when $V_{x+k}^{(k)}=V_{x+k}^{(k-1)}$, the health benefit and surrender value increases are completely financed by the policyholder via increased premium payments. In this special case we find from (18) and (20) that

$$
\pi^{(k)}-\pi^{(k-1)}=\frac{\left(B_{x+k}^{(k)}-B_{x+k}^{(k-1)}\right)+\left(W_{x+k}^{(k)}-W_{x+k}^{(k-1)}\right)}{\ddot{a}_{x+k}^{a a}} .
$$

This means that the premium increase $\pi^{(k)}-\pi^{(k-1)}$ introduced at time $k$ can be interpreted as the level premium for an insurance contract with yearly benefits equal to the health benefit increases and with surrender values equal to the surrender value increases of the original contract.

\subsection{Generalizing the recursions: from one-step to $j$-step}

We develop additional insights in the reserve calculations by generalizing some of the one-step recursions developed in Sections 3.1 and 3.2. These will be useful in the next section. Starting from the available reserve $V_{x+k}^{(k)}$ at time $k$, we introduce the notation $V_{x+k+j+1}^{(k)}$ for the available reserve of the contract at time $k+j+1$ in case of a future yearly medical inflation of $f$ in the interval $(k, k+j+1)$ as predicted at time $k$. Assuming the technical basis at time $k$, the available reserve follows from the forward recursion

$$
V_{x+k+j+1}^{(k)}=\left(V_{x+k+j}^{(k)}+\pi^{(k)}-b_{x+k+j}^{(k)}-q_{x+k+j}^{a w} v w_{x+k+j+1}^{(k)}\right)\left(p_{x+k+j}^{a a} v\right)^{-1},
$$

or equivalently,

$$
\begin{aligned}
v V_{x+k+j+1}^{(k)}= & V_{x+k+j}^{(k)}+\pi^{(k)}-b_{x+k+j}^{(k)}+q_{x+k+j}^{a d} v V_{x+k+j+1}^{(k)} \\
& -q_{x+k+j}^{a w} v\left(w_{x+k+j+1}^{(k)}-V_{x+k+j+1}^{(k)}\right)
\end{aligned}
$$

which holds for $j \in\{0,1, \ldots, \omega-x-k-1\}$. The initial value $V_{x+k}^{(k)}$ is given by (20), where $V_{x}^{(0)}=0$.

It is easy to verify that the solution of recursion (23), with initial value $V_{x+k}^{(k)}$, can be expressed in the following retrospective form

$$
\begin{aligned}
V_{x+k+j}^{(k)}= & V_{x+k}^{(k)}\left({ }_{j} p_{x+k}^{a a} v^{j}\right)^{-1} \\
& +\sum_{l=0}^{j-1}\left(\pi^{(k)}-b_{x+k+l}^{(k)}-q_{x+k+l}^{a w} v w_{x+k+l+1}^{(k)}\right)\left({ }_{j-l} p_{x+k+l}^{a a} v^{j-l}\right)^{-1}
\end{aligned}
$$

for $j \in\{0,1, \ldots, \omega-x-k\}$. Taking into account the restored actuarial equivalence (20), the reserves $V_{x+k+j}^{(k)}$ can also be expressed prospectively

$$
V_{x+k+j}^{(k)}=B_{x+k+j}^{(k)}+W_{x+k+j}^{(k)}-\pi^{(k)} \ddot{a}_{x+k+j}^{a a},
$$

with $\ddot{a}_{x+k+j}^{a a}, B_{x+k+j}^{(k)}$ and $W_{x+k+j}^{(k)}$ defined in (7), (11) and (12), respectively. In particular, we find that

$$
V_{\omega}^{(k)}=b_{\omega}^{(k)}-\pi^{(k)}
$$

which means that the available reserve at the last attainable integer age is equal to the health benefit minus the premium to be be paid at that time. 


\subsection{Discussion}

Our technical basis includes a constant yearly technical interest rate $i$ and a constant and ageindependent yearly medical inflation $f$. However, all results can be generalized to include varying deterministic technical interest rates and varying deterministic yearly medical inflation rates.

The methodology explained in this paper can be used in very different situations, depending on the elements of the technical basis that are guaranteed and those elements that are subject to revision according to policy conditions. We illustrate these situations with two extreme examples.

Consider first a health insurance contract where no element of the technical basis is contractually guaranteed. In this case, the contract is similar to a pooling agreement. The contract is now updated at time $k$, starting from the recursion (13), but $b_{x+k-1}^{(k-1)}, q_{x+k-1}^{a w}, v$ and $p_{x+k-1}^{a a}$ correspond to observed values over the past year. Premium and reserve are then updated according to (20), for some appropriately chosen technical basis for the required reserve at time $k$.

Second, consider the situation where the policy guarantees the technical basis, except for the health benefits. In this case, the contractual reserve at time $k, V_{x+k}^{(k-1)}$, is determined from (13), where the $q_{x+k-1}^{a w}, v$ and $p_{x+k-1}^{a a}$ are those specified in the contract. But it may happen that the accumulated assets for the contract differ from the contractual reserve $V_{x+k}^{(k-1)}$. If the guaranteed technical basis turns out to be conservative, accumulated assets exceed the contractual reserve, resulting in technical profits. The contract can then be updated according to (20), where $V_{x+k}^{(k)}$ is the sum of $V_{x+k}^{(k-1)}$ and the participating gain awarded at time $k$. This profit sharing mechanism alters the new premium level $\pi^{(k)}$.

Besides these two extreme cases, intermediate cases, where e.g. mortality and interest are guaranteed, but inflation and lapse rates are not, may of course be considered in the same updating framework as developed in this paper.

\section{Defining the surrender values}

This section designs and investigates two possible specifications for the surrender values in the lifelong health insurance contract. For both specifications we calculate the initial premium and show explicitly how to solve the actuarial equivalence.

\subsection{Reserve-dependent surrender values}

We consider the case where upon surrender in year $(k, k+1)$ the surrender value that is paid out at time $k+1$ is a linear function of the available reserve

$$
w_{x+k+1}^{(k)}=\left(1-\beta_{k+1}\right) V_{x+k+1}^{(k)}-\alpha_{k+1}, \quad k=0,1,2, \ldots, \omega-x-1,
$$

with $w_{\omega+1}^{(0)}$ equal to zero, $\alpha_{k+1} \geq 0$ a reserve-independent penalty and $0<\beta_{k+1} \leq 1$ the nontransferred percentage of the available reserve in case of surrender. The quantities $\alpha_{k+1}$ and $\beta_{k+1}$ are fixed at policy issue. Benefits of the form (28) have been studied in a continuous-time setting by Christiansen et al. (2014), without allowance for medical inflation. The reserve $V_{x+k+1}^{(k)}$ is a 
function of $\pi^{(0)}, \pi^{(1)}, \ldots, \pi^{(k)}$. Therefore, at policy issue, this reserve and hence, the surrender value $w_{x+k+1}^{(k)}$, are in general unknown. However, when the surrender option is exercised in year $(k, k+1)$, the reserve $V_{x+k+1}^{(k)}$ and the surrender value are known at time $k+1$, see (13).

Initial premium. Let us first determine the level premium $\pi^{(0)}$ at policy issue. In order to be able to determine this premium from the equivalence principle (10), we choose time 0 observable values for the future surrender values. We propose to estimate the surrender value in case of surrender in year $(j, j+1), j \in\{0,1,2, \ldots, \omega-x-1\}$, by

$$
w_{x+j+1}^{(0)}=\left(1-\beta_{j+1}\right) V_{x+j+1}^{(0)}-\alpha_{j+1},
$$

where $V_{x+j+1}^{(0)}$ is the estimate for the available reserve at time $j+1$ defined by the recursion (23), with initial value $V_{x}^{(0)}=0$.

Taking into account that the reserves (and hence, also $W_{x}^{(0)}$ ) depend on the premium $\pi^{(0)}$, the equivalence relation (10) does not lead to an explicit expression for $\pi^{(0)}$. In order to find such an explicit expression, we insert the surrender values (29) into recursion (23). Re-arranging the terms in this recursion leads to the transformed expression

$$
V_{x+j+1}^{(0)}=\left(V_{x+j}^{(0)}+\pi^{(0)}-b_{x+j}^{(0)}-\bar{q}_{x+j}^{a w} v \bar{w}_{x+j+1}\right)\left(\bar{p}_{x+j}^{a a} v\right)^{-1},
$$

which holds for any $j \in\{0,1, \ldots, \omega-x-1\}$, still with initial value $V_{x}^{(0)}=0$, where

$$
\bar{q}_{x+j}^{a w}=\beta_{j+1} q_{x+j}^{a w}, \quad \bar{w}_{x+j+1}=-\frac{\alpha_{j+1}}{\beta_{j+1}} \quad \text { and } \quad \bar{p}_{x+j}^{a a}=1-q_{x+j}^{a d}-\bar{q}_{x+j}^{a w} .
$$

Furthermore, we set

$$
\bar{q}_{\omega}^{a w}=0 \text { and } \bar{w}_{\omega+1}=0 .
$$

As $\beta_{j+1} \in(0,1]$, we have $\bar{q}_{x+j}^{a w} \in[0,1]$ so that this quantity and $\bar{p}_{x+j}^{a a}$ can be interpreted as probabilities.

We can conclude that, at any time $j+1$, the reserve $V_{x+j+1}^{(0)}$ of the health insurance contract, defined by recursion (23) with initial value $V_{x}^{(0)}=0$, is identical to the reserve of an artificial health insurance contract with transformed surrender values $\bar{w}_{x+j+1}$ and transformed probabilities $\bar{q}_{x+j}^{a w}$ and $\bar{p}_{x+j}^{a a}$. The reserves of this artificial contract follow from recursion (30) with initial value $V_{x}^{(0)}=0$. In the following proposition, we derive an explicit expression for the initial premium of the original contract.

Proposition 1. An explicit expression for the initial premium $\pi^{(0)}$ of the health insurance contract with reserve-dependent surrender values (29) is given by

$$
\pi^{(0)}=\frac{\underline{B}_{x}^{(0)}+\underline{W}_{x}^{(0)}}{\underline{\ddot{a}}_{x}^{a a}},
$$


with

$$
\begin{aligned}
\underline{B}_{x}^{(0)} & =\sum_{l=0}^{\omega-x} l \bar{p}_{x}^{a a} v^{l} b_{x+l}^{(0)} \\
\underline{W}_{x}^{(0)} & =\sum_{l=0}^{\omega-x} l \bar{p}_{x}^{a a} \bar{q}_{x+l}^{a w} v^{l+1} \bar{w}_{x+l+1} \\
\underline{a}_{x}^{a a} & =\sum_{l=0}^{\omega-x} l \bar{p}_{x}^{a a} v^{l} .
\end{aligned}
$$

In these expressions, the $\bar{q}_{x+l}^{a w}$ and $\bar{w}_{x+l+1}$ are defined by (31) and (32). Furthermore, ${ }_{0} \bar{p}_{x+j}^{a a}=1$ and for $l>0$, we have

$$
{ }_{l} \bar{p}_{x}^{a a}=\prod_{k=0}^{l-1} \bar{p}_{x+k}^{a a}
$$

with the $\bar{p}_{x+k}^{a a}$ defined in (31).

Proof. Following Section 3.4, the recursion (23) for $k=0$ with initial value $V_{x}^{(0)}=0$ leads to the retrospective expression (25) with $j=\omega-x$ to obtain $V_{\omega}^{(0)}$. In a similar way, the transformed version (30) of this recursion with the same initial value leads to the following expression for $V_{\omega}^{(0)}$ :

$$
V_{\omega}^{(0)}=\sum_{l=0}^{\omega-x-1}\left(\pi^{(0)}-b_{x+l}^{(0)}-\bar{q}_{x+l}^{a w} v \bar{w}_{x+l+1}\right)\left({ }_{\omega-x-l} \bar{p}_{x+l}^{a a} v^{\omega-x-l}\right)^{-1} .
$$

On the other hand, from (27) we know that $V_{\omega}^{(0)}=b_{\omega}^{(0)}-\pi^{(0)}$. Hence,

$$
\sum_{l=0}^{\omega-x}\left(\pi^{(0)}-b_{x+l}^{(0)}-\bar{q}_{x+l}^{a w} v \bar{w}_{x+l+1}\right)\left({ }_{\omega-x-l} \bar{p}_{x+l}^{a a} v^{\omega-x-l}\right)^{-1}=0 .
$$

Multiplying each term in this expression by ${ }_{\omega-x} \bar{p}_{x}^{a a} v^{\omega-x}$ leads to

$$
\pi^{(0)} \underline{\ddot{a}}_{x}^{a a}=\underline{B}_{x}^{(0)}+\underline{W}_{x}^{(0)},
$$

which proves the stated result.

Obviously, $\underline{B}_{x}^{(0)}$ and $\underline{W}_{x}^{(0)}$ can directly be determined at policy issue. This means that (33) is indeed an explicit expression for the initial premium.

Updating mechanism. Suppose now that we have arrived at time $k \in\{1,2, \ldots, \omega-x\}$, and that the contract is still in force. The actuarial value of future health benefit payments, taking into account medical inflation up to that time, is then given by $B_{x+k}^{(k)}$, which is defined in (11). As before, the updated value of the available reserve at time $k$ is denoted by $V_{x+k}^{(k)}$, whereas the new level premium to be determined at that time is denoted by $\pi^{(k)}$. We propose to update the surrender values at time $k$ using the information about observed inflation until time $k$, and assuming a yearly medical inflation of $f$ for future years:

$$
w_{x+k+j+1}^{(k)}=\left(1-\beta_{k+j+1}\right) V_{x+k+j+1}^{(k)}-\alpha_{k+j+1}, \quad j=0,1, \ldots, \omega-x-k-1,
$$


where $V_{x+k+j+1}^{(k)}$ is the available reserve at time $k+j+1$ as defined in (23).

Taking into account that the reserves $V_{x+k+j+1}^{(k)}$ and hence also $W_{x+k}^{(k)}$, depend on the premium $\pi^{(k)}$, we find that restoring equivalence equation (20) does not give an explicit relation between the updated premium and the available reserve at time $k$. In order to solve this problem, we insert the values (34) for the updated surrender values in the recursion (23). This leads to the transformed recursion for the available reserves:

$$
V_{x+k+j+1}^{(k)}=\left(V_{x+k+j}^{(k)}+\pi^{(k)}-b_{x+k+j}^{(k)}-\bar{q}_{x+k+j}^{a w} v \bar{w}_{x+k+j+1}\right)\left(\bar{p}_{x+k+j}^{a a} v\right)^{-1},
$$

which holds for any $j \in\{0,1, \ldots, \omega-x-k-1\}$, with initial value $V_{x+k}^{(k)}$. The quantities $\bar{q}_{x+k+j}^{a w}$, $\bar{p}_{x+k+j}^{a a}$ and $\bar{w}_{x+k+j+1}$ are defined as before. Rewriting the recursion this way allows us to find an explicit relation between $V_{x+k}^{(k)}$ and $\pi^{(k)}$, as is shown in the following proposition.

Proposition 2. Consider the lifelong health insurance contract with reserve-dependent surrender values (28). Relation (20) at time $k$ can be expressed in the following way:

$$
V_{x+k}^{(k)}=\underline{B}_{x+k}^{(k)}+\underline{W}_{x+k}^{(k)}-\pi^{(k)} \underline{\ddot{a}}_{x+k}^{a a},
$$

with

$$
\begin{aligned}
\underline{B}_{x+k}^{(k)} & =\sum_{l=0}^{\omega-x-k} l_{x+k}^{a a} v^{l} b_{x+k+l}^{(k)} \\
\underline{W}_{x+k}^{(k)} & =\sum_{l=0}^{\omega-x-k} l \bar{p}_{x+k}^{a a} \bar{q}_{x+k+l}^{a w} v^{l+1} \bar{w}_{x+k+l+1} \\
\ddot{a}_{x+k}^{a a} & =\sum_{l=0}^{\omega-x-k} l \bar{p}_{x+k}^{a a} v^{l} .
\end{aligned}
$$

In these expresssions, the $\bar{q}_{x+k+l}^{a w}, l_{p+k}^{a a}$ and $\bar{w}_{x+k+l+1}$ are defined as in Proposition 1.

Proof. Following Section 3.4, the recursion (23) with initial value $V_{x+k}^{(k)}$ for the available reserves leads to the retrospective expression (25) with $j=\omega-x-k$ to obtain $V_{\omega}^{(0)}$. The proof here follows a similar reasoning as in Proposition 1.

This result makes restoring the actuarial equivalence as discussed in Section 3 easy.

\subsection{Premium-dependent surrender values}

Actuaries generally base surrender values on accumulated available reserves and this case has been thoroughly investigated in Section 4.1. However, this concept may seem obscure to many policyholders. Moreover, some insurers do not compute individual reserves but rather manage the entire portfolio as a collective. In order to overcome these concerns, one might prefer to consider surrender values based on the premiums paid up to the moment of surrender. In 
particular, we assume that in case of surrender in the year $(k, k+1)$, the surrender value paid out at time $k+1$ is given by

$$
w_{x+k+1}^{(k)}=\beta_{k+1} \sum_{l=0}^{k} \pi^{(l)}\left(1+i^{\prime}\right)^{k+1-l}-\alpha_{k+1}, \quad k=0,1,2, \ldots
$$

Hence, the surrender value is equal to a time-dependent fraction $\beta_{k+1}, 0 \leq \beta_{k+1} \leq 1$, of the accumulated value of the premiums paid until time $k$, minus a time-dependent penalty $\alpha_{k+1} \geq 0$. We assume that the quantities $\beta_{1}, \beta_{2}, \ldots$ and $\alpha_{1}, \alpha_{2}, \ldots$ are fixed at policy issue. The coefficients $\beta_{1}, \beta_{2}, \ldots$ can be chosen such that they approximately mimic the accumulation of the savings premiums in the reserve, representing the part of the premiums paid but not consumed to finance past health benefits. The accumulation of the premiums is performed at a constant interest rate $i^{\prime}$, which may be different from the technical interest rate $i$. We could for instance set $i^{\prime}=0$ so that premiums enter the calculation at nominal values.

Initial premium. At policy issue, the payment for surrender in year $(k, k+1)$ is in general unknown as it depends on the a priori unknown stream of future premium payments $\pi^{(1)}, \pi^{(2)}, \ldots, \pi^{(k)}$. However, when the surrender option is exercised in year $(k, k+1)$, the surrender value $w_{x+k+1}^{(k)}$ that is actually paid out is fully specified at time $k+1$, based on the information that is available at that time about previous medical inflation.

In order to be able to determine the initial level premium $\pi^{(0)}$ from the equivalence principle (10), we have to choose values for the future surrender values that are observable at time 0 . We propose to estimate the payment in case of surrender in year $(j, j+1), j \in\{0,1,2, \ldots, \omega-x-1\}$, by

$$
w_{x+j+1}^{(0)}=\beta_{j+1} \sum_{l=0}^{j} \pi^{(0)}\left(1+i^{\prime}\right)^{j+1-l}-\alpha_{j+1} .
$$

This means that $w_{x+j+1}^{(0)}$ corresponds to the surrender value $w_{x+j+1}^{(j)}$ in case of a medical inflation of $f$ per year and no updating of the premiums until surrender.

Proposition 3. Assuming (38), an explicit expression for the initial premium $\pi^{(0)}$ of the health insurance contract with premium-dependent surrender benefits (37) is given by

$$
\pi^{(0)}=\frac{B_{x}^{(0)}-\sum_{j=0}^{\omega-x}{ }_{j} p_{x}^{a a} q_{x+j}^{a w} v^{j+1} \alpha_{j+1}}{\ddot{a}_{x}^{a a}-\sum_{j=0}^{\omega-x}{ }_{j} p_{x}^{a a} q_{x+j}^{a w} v^{j+1} c_{j+1}^{(0)}}
$$

with

$$
c_{j+1}^{(0)}=\beta_{j+1} \sum_{l=0}^{j}\left(1+i^{\prime}\right)^{j+1-l} .
$$

Proof. We rewrite (38) as follows:

$$
w_{x+j+1}^{(0)}=c_{j+1}^{(0)} \pi^{(0)}-\alpha_{j+1}
$$

with the $c_{j+1}^{(0)}$ defined in (40). Inserting the surrender values $w_{x+j+1}^{(0)}$ in the actuarial equivalence relation (10), while taking into account (12), leads to the explicit expression (39) for the initial level premium $\pi^{(0)}$. 
Updating mechanism. Suppose now that we have arrived at time $k \in\{1,2, \ldots\}$ and that the policy is still in force. The available reserve $V_{x+k}^{(k-1)}$ at this moment is given by (13). Taking into account the information about medical inflation up to time $k$, the future health benefits are re-estimated and their updated actuarial value $B_{x+k}^{(k)}$ follows from (11). The previously chosen values $w_{x+k+j+1}^{(k-1)}$ for future surrender values are replaced by the values $w_{x+k+j+1}^{(k)}$, which are defined by

$$
w_{x+k+j+1}^{(k)}=\beta_{k+j+1} \sum_{l=0}^{k+j} \pi^{(\min \{k, l\})}\left(1+i^{\prime}\right)^{k+j+1-l}-\alpha_{k+j+1} .
$$

At time $k$, the future surrender values are determined using the information about medical inflation until time $k$, while assuming a future inflation of $f$ per year. To restore the actuarial equivalence at time $k$, the insurer updates the available reserve to level $V_{x+k}^{(k)}$ and the premium is replaced by $\pi^{(k)}$.

Proposition 4. Consider the lifelong health insurance contract with premium-dependent surrender values (37). Relation (20) can be expressed as:

$$
\begin{aligned}
V_{x+k}^{(k)}= & B_{x+k}^{(k)}+\sum_{j=0}^{\omega-x-k}{ }_{j} p_{x+k}^{a a} q_{x+k+j}^{a w} v^{j+1} d_{k+j+1}^{(k)} \\
& -\pi^{(k)}\left(\ddot{a}_{x+k}^{a a}-\sum_{j=0}^{\omega-x-k}{ }_{j} p_{x+k}^{a a} q_{x+k+j}^{a w} v^{j+1} c_{k+j+1}^{(k)}\right)
\end{aligned}
$$

with

$$
\begin{aligned}
c_{k+j+1}^{(k)} & =\beta_{k+j+1} \sum_{l=k}^{k+j}\left(1+i^{\prime}\right)^{k+j+1-l} \\
d_{k+j+1}^{(k)} & =\beta_{k+j+1} \sum_{l=0}^{k-1} \pi^{(l)}\left(1+i^{\prime}\right)^{k+j+1-l}-\alpha_{k+j+1} .
\end{aligned}
$$

Proof. The updated surrender value $w_{x+k+j+1}^{(k)}$ can be rewritten as

$$
w_{x+k+j+1}^{(k)}=c_{k+j+1}^{(k)} \pi^{(k)}+d_{k+j+1}^{(k)},
$$

with $c_{k+j+1}^{(k)}$ and $d_{k+j+1}^{(k)}$ defined by (44) and (45), respectively. Substituting (46) into (12) and combining with (20) yields the announced result.

The explicit relation between $V_{x+k}^{(k)}$ and $\pi^{(k)}$ in Proposition 4 allows to restore the actuarial equivalence (20) in a straightforward way.

\section{$5 \quad$ Numerical illustrations}

\subsection{Contract and technical basis}

We consider a contract issued to a policyholder aged $x=25$. This contract covers medical expenses in excess of social security, as those commonly sold in Belgium. Additional background 
information is in Devolder et al. (2008). The technical basis assumes a yearly interest rate $i$ of $2 \%$.

The dashed line in Figure 2 shows the average health benefit $\bar{b}_{y}^{(0)}$ as a function of the policyholder's attained age $y$, in euros. The shape of $y \mapsto \bar{b}_{y}^{(0)}$ is inspired by Belgian market experience on private health insurance covers but the values have been rescaled for confidentiality reasons. The full line in this figure represents the average health benefits $b_{y}^{(0)}$, given by (1), when a medical inflation $f$ of $2 \%$ per year is taken into account.

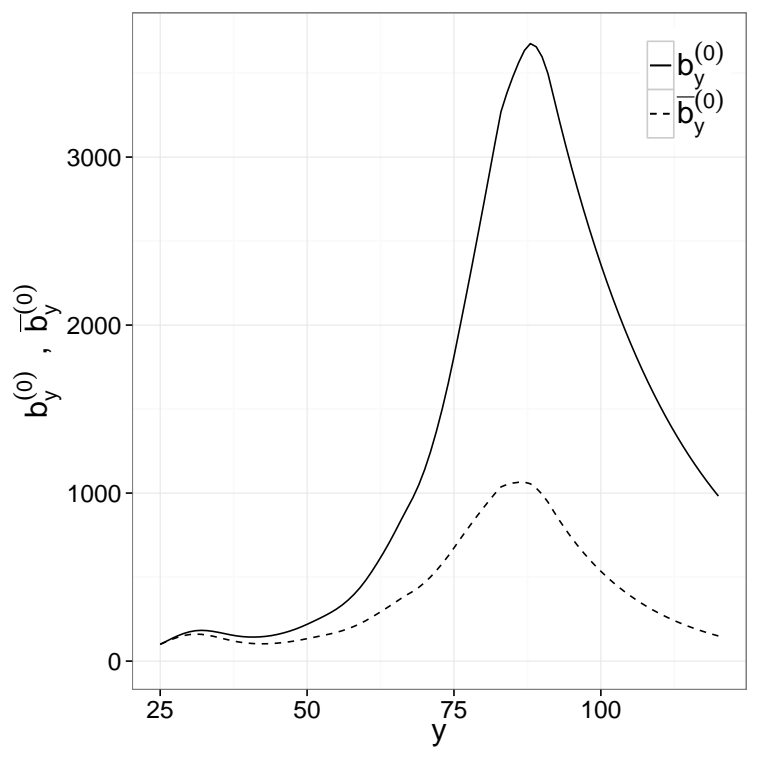

Figure 2: Average health benefit $\bar{b}_{y}^{(0)}$ at age $y$ (dashed line) and inflated values $b_{y}^{(0)}$ (full line) at constant rate $f$ for medical inflation.

Since health insurance contracts with a transferable reserve are not currently available on the Belgian market, we do not have relevant observed lapse probabilities at our disposal. Therefore we carry out a sensitivity analysis by varying the lapse probabilities according to the following three scenarios: for a policyholder buying the contract at age 25, we consider one-year lapse probabilities $q_{y}^{a w}$ at age $y \geq 25$ given by

$$
\begin{aligned}
& q_{y}^{a w_{1}}=0 \\
& q_{y}^{a w_{2}}= \begin{cases}0.1-0.002 \cdot(y-20) & \text { if } 25 \leq y \leq 70 \\
0 & \text { otherwise }\end{cases} \\
& q_{y}^{a w_{3}}= \begin{cases}0.05 \cdot\left(\cos \left((y-25) \cdot \frac{\pi}{95}\right)+1\right) & \text { if } 25 \leq y \leq 120 \\
0 & \text { otherwise. }\end{cases}
\end{aligned}
$$

These lapse probabilities are displayed in Figure 3. Under the first set, $q_{y}^{a w_{1}}$, policyholders never cancel the contract. The second set of lapse probabilities $q_{y}^{a w_{2}}$ has been used by Vercruysse et al. (2013). These probabilities imply a higher tendency to lapse at younger ages for the 25-year old policyholder under consideration, but no lapse after age 70. This is often taken as the central scenario on the Belgian market. Finally, under the third set of lapse probabilities, $q_{y}^{a w_{3}}$, we have higher lapse probabilities at younger ages which then decline smoothly to 0 at the ultimate age $\omega=120$. 


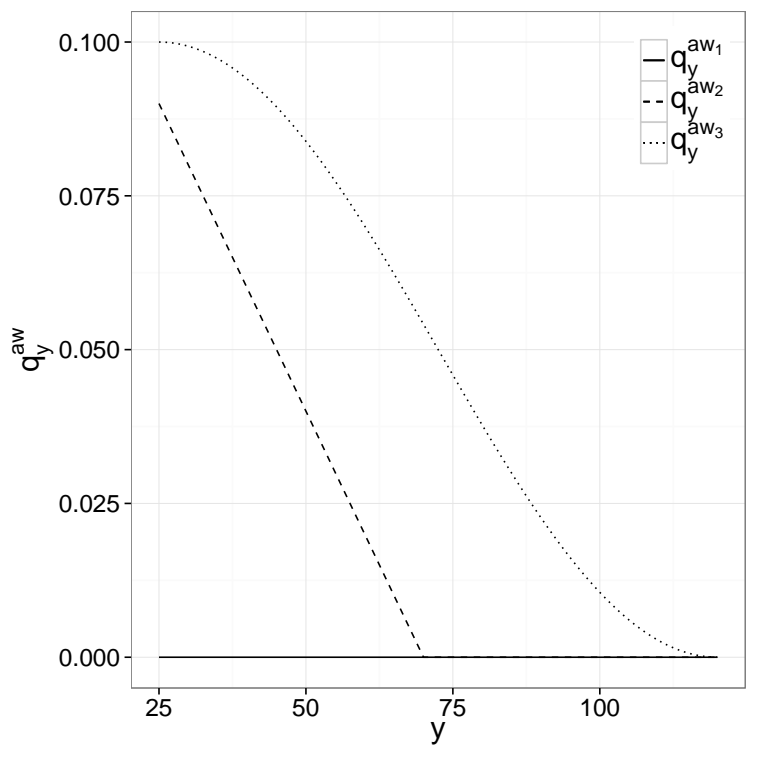

Figure 3: One-year lapse probabilities $q_{y}^{a w_{1}}$ (full line), $q_{y}^{a w_{2}}$ (dashed line), and $q_{y}^{a w_{3}}$ (dotted line).

Death probabilities are displayed in Figure 4, left panel. Notice that these are not the $q_{y}^{\text {ad }}$ but yearly death probabilities $q_{y}^{\prime d}$ based on observations at the general population level in a single decrement, two state, 'alive or dead' model. We recover the values for $q_{y}^{a d}$ from the following relation

$$
q_{y}^{a d}=q_{y}^{\prime d}\left(1-\frac{q_{y}^{a w}}{2-q_{y}^{\prime d}}\right) .
$$

This relation holds under the assumption of a uniform distribution of decrements in each of the corresponding single decrement models ('alive vs withdrawn' and 'alive vs dead'; see Section 8.10.2 in Dickson et al. (2013)). Notice that lapse probabilities enter the calculation of death probabilities so that different lapse scenarios have impact on all actuarial quantities. Under (47), we have $q_{y}^{a d}=q_{y}^{\prime d}$. Figure 4, right panel, displays the three sets of one-year death probabilities used in the numerical illustrations.

\subsection{Surrender values}

\subsubsection{Reserve-dependent surrender values}

When surrender values depend on the available reserve, as discussed in Section 4.1, we specify the non-transferred percentage and the reserve-independent penalty in the definition of surrender values (28) as follows

$$
\beta_{k+1}=\left\{\begin{array}{ll}
1 & \text { if } 0 \leq k \leq 4 \\
0.2 & \text { if } 5 \leq k
\end{array} \quad \text { and } \quad \alpha_{k+1}= \begin{cases}0 & \text { if } 0 \leq k \leq 4 \\
150 & \text { if } 5 \leq k .\end{cases}\right.
$$

Early cancellations often cause significant losses for the insurer due to unrecovered administrative costs and commissions. Therefore, no surrender values are paid in the first five years of the contract in this example. Afterwards we fix the non-transferred percentage at $20 \%$ and take the reserve-independent penalty equal to 150 . 

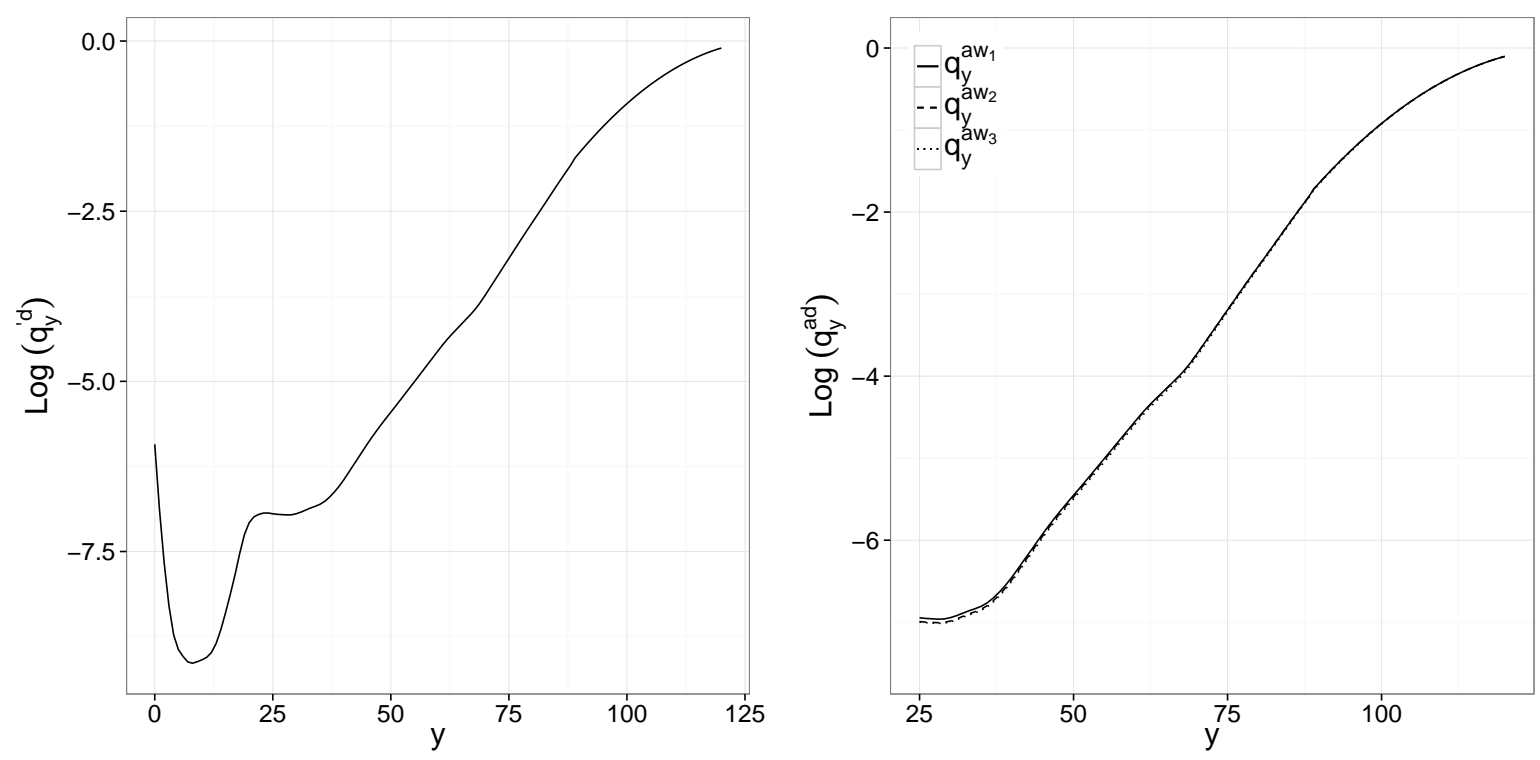

Figure 4: One-year death probabilities $q_{y}^{\prime d}$ on a log-scale (left) and one-year death probabilities $q_{y}^{a d}$ on a $\log$-scale corresponding to the three sets of lapse probabilities (right) $q_{y}^{a w_{1}}$ (full line), $q_{y}^{a w_{2}}$ (dashed line), and $q_{y}^{a w_{3}}$ (dotted line).

\subsubsection{Premium-dependent surrender values}

We also consider surrender values based on the premiums paid so far, as discussed in Section 4.2. We set the interest rate on the accumulated premiums equal to $i^{\prime}=1 \%$.

We propose to specify the time-dependent fractions $\beta_{k}$ and time-dependent penalties $\alpha_{k}$ in (37) as follows in the policy conditions:

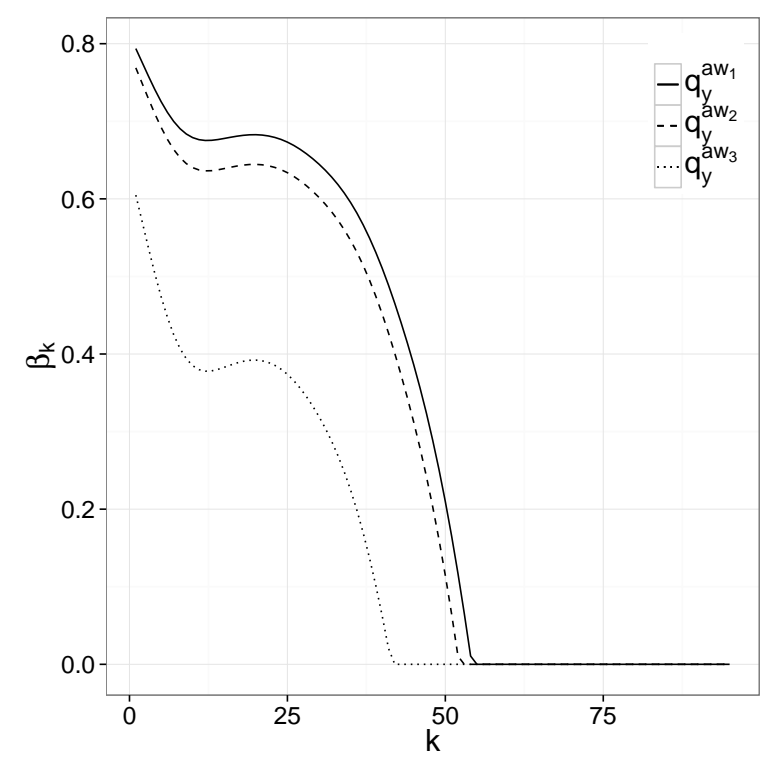

Figure 5: Values of the $\beta_{k}$ defined in (51). 
1. Define $\beta_{k}^{\prime}=1$ and $\alpha_{k}^{\prime}=\sum_{l=0}^{k-1} b_{x+l}^{(0)} \cdot\left(1+i^{\prime}\right)^{k-l}$. A contract with surrender benefits defined by (37) with parameters $\beta_{k}^{\prime}$ and $\alpha_{k}^{\prime}$ defines surrender benefits at time $k+1$ as the sum of the savings premiums at policy issue with interest accumulation at rate $i^{\prime}$ :

$$
w_{x+k+1}^{\prime(0)}=\sum_{l=0}^{k}\left(\pi^{(0)}-b_{x+l}^{(0)}\right) \cdot\left(1+i^{\prime}\right)^{k+1-l} .
$$

2. Calculate the initial premium $\pi^{\prime(0)}$ for a contract with surrender values as defined in step 1. Use formula (39) from the approach outlined in Section 4.2.

3. Define

$$
\beta_{k}=\max \left\{0, \frac{\sum_{l=0}^{k-1}\left(\pi^{\prime}(0)-b_{x+l}^{(0)}\right) \cdot\left(1+i^{\prime}\right)^{k-l}}{\sum_{l=0}^{k-1} \pi^{\prime}(0) \cdot\left(1+i^{\prime}\right)^{k-l}}\right\}
$$

and $\alpha_{k}=0$ which leads to surrender values

$$
w_{x+k+1}^{(0)}=\max \left\{0, \frac{\sum_{l=0}^{k}\left(\pi^{\prime}(0)-b_{x+l}^{(0)}\right) \cdot\left(1+i^{\prime}\right)^{k+1-l}}{\sum_{l=0}^{k} \pi^{\prime}(0) \cdot\left(1+i^{\prime}\right)^{k+1-l}}\right\} \cdot \sum_{l=0}^{k} \pi^{(0)}\left(1+i^{\prime}\right)^{k+1-l} .
$$

These three simple steps base the definition of the surrender values on the sum of the savings premiums and ensure that the surrender values do not become negative. Moreover, this definition also ensures that the surrender values never exceed the sum of the premiums paid so far with interest accumulated at rate $i^{\prime}$ since $\beta_{k} \leq 1$. Figure 5 displays the $\beta_{k}$ as specified in (51).

\subsection{Initial premium}

Table 1 shows the initial premium $\pi^{(0)}$ calculated for different types of surrender values: reservedependent (Section 4.1), premium-dependent (Section 4.2), and no surrender value (i.e. the policyholder does not receive any benefit in case of surrender). Obviously, the initial premium is identical under the first set of lapse probabilities $q_{y}^{a w_{1}}$ as the definition of the surrender values is irrelevant in that case. The last column of Table 1 shows the initial premium in case the surrender values are always 0 . Table 1 illustrates that, given any non-zero lapse probability, a higher surrender value increases the initial premium.

\begin{tabular}{l|lll}
\hline Type of surrender values & Reserve-dependent & Premium-dependent & No payment \\
\hline Lapse probability $q_{y}^{a w_{1}}$ & 484.76 & 484.76 & 484.76 \\
Lapse probability $q_{y}^{a w_{2}}$ & 415.50 & 431.15 & 267.18 \\
Lapse probability $q_{y}^{a w_{3}}$ & 238.90 & 256.15 & 140.50 \\
\hline
\end{tabular}

Table 1: Premium $\pi^{(0)}$ at policy issue with three different specifications for the surrender value: reservedependent as in Section 4.1, premium-dependent as in Section 4.2 and a surrender value equal to zero. 


\subsection{Medical inflation scenario}

We illustrate the proposed methods by assuming an additional yearly medical inflation $j_{k}^{[B]}=1 \%$ for health benefits, on top of the expected inflation $f=2 \%$ incorporated in the premiums. In the notation of Section 2.1, the assumption of the expected inflation of $f=2 \%$ translates to

$$
b_{x+j}^{(0)}=(1+f)^{j} \cdot \bar{b}_{x+j}^{(0)}=(1+2 \%)^{j} \cdot \bar{b}_{x+j}^{(0)} .
$$

The assumption of the additional medical inflation can be written as (with $k \geq 1$ )

$$
\bar{b}_{x+k+j}^{(k)}=(1+f) \cdot\left(1+j_{k}^{[B]}\right) \cdot \bar{b}_{x+k+j}^{(k-1)}
$$

such that

$$
b_{x+k+j}^{(k)}=(1+f)^{j} \cdot \bar{b}_{x+k+j}^{(k)}=(1+2 \%)^{j} \cdot \bar{b}_{x+k+j}^{(k)}
$$

or

$$
b_{x+k+j}^{(k)}=\left(1+j_{k}^{[B]}\right) \cdot b_{x+k+j}^{(k-1)}=(1+1 \%) \cdot b_{x+k+j}^{(k-1)} .
$$

\subsection{Updating mechanisms}

As explained earlier, many ways exist to update the contract to account for medical inflation. As in Vercruysse et al. (2013) we denote the premium and the reserve indexing at time $k$ with $j_{k}^{[P]}$ and $j_{k}^{[V]}$, respectively. We consider the following two updating mechanisms.

Mechanism 1: The premium index $j_{k}^{[P]}$ is contractually fixed as a function of the observed medical inflation, i.e. $j_{k}^{[P]}=j_{k}^{[P]}\left(j_{k}^{[B]}\right)$. Premium updates are then obtained from

$$
\pi^{(k)}=\left(1+j_{k}^{[P]}\left(j_{k}^{[B]}\right)\right) \cdot \pi_{x}^{(k-1)} .
$$

The reserves are adjusted afterwards. For instance, policy conditions could specify that premiums are updated according to

$$
j_{k}^{[P]}\left(j_{k}^{[B]}\right)=(1+\gamma) \cdot j_{k}^{[B]}
$$

where the $\gamma$ accounts for the indexing of the accumulated reserve. In the numerical illustration in Sections 5.6, 5.7 and 5.8 we consider (58) with $\gamma=0$.

Mechanism 2: The insurer now first increases the available reserve according to

$$
V_{x+k}^{(k)}=\left(1+j_{k}^{[V]}\right) \cdot V_{x+k}^{(k-1)}
$$

and determines the corresponding new level premium afterwards. We illustrate this updating mechanism for $j_{k}^{[V]}=1 \%$, i.e. the insurer increases the available reserve by $1 \%$ each year. 


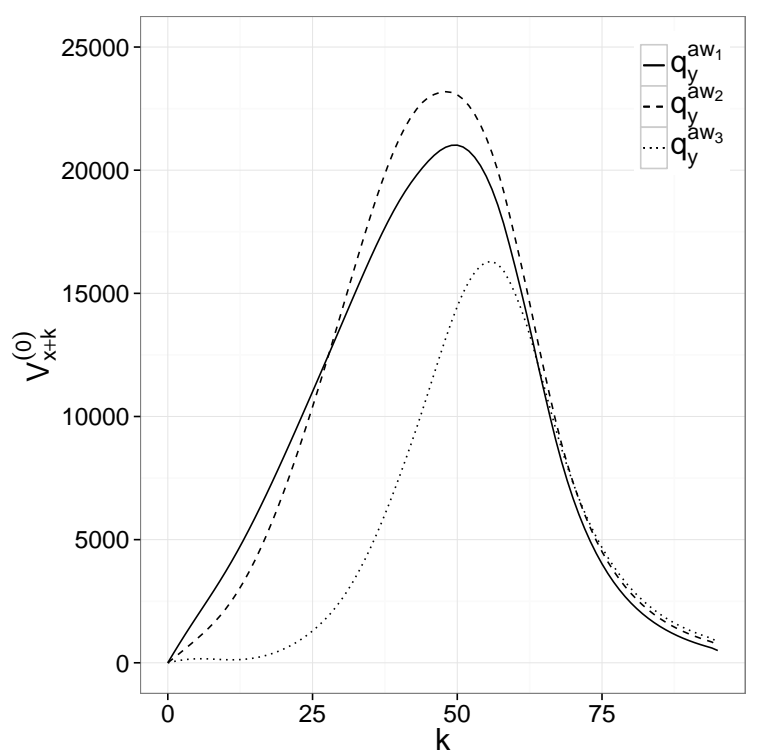

Figure 6: No surrender value: available reserves $V_{x+k}^{(0)}$ as calculated with the different types of lapse probabilities $q_{y}^{a w_{1}}$ (full line), $q_{y}^{a w_{2}}$ (dashed line), $q_{y}^{a w_{3}}$ (dotted line).

\subsection{The case of no surrender values}

We start with the simple setting from Vercruysse et al. (2013) where the policyholder receives no surrender value in case of surrender. Figure 6 shows the available reserves calculated with information available at time 0 . Higher lapse probabilities lead to a lower premium and, as a consequence, tend to decrease the reserve early in the policy. The reserve not paid out as a surrender value to a lapsing policyholder is used to cover this.

Figure 7 illustrates the evolution of the reserves $V_{x+k}^{(k)}$ and the premiums $\pi^{(k)}$ over time when nothing is paid in case of surrender. In absence of surrender values, the result of updating mechanisms 1 and 2 is exactly the same. This is explained from the fact that the expected present value of the surrender values (12) is zero which reduces actuarial equivalence (18) at time $(k-1)$ to

$$
V_{x+k}^{(k-1)}=B_{x+k}^{(k-1)}-\pi^{(k-1)} \cdot \ddot{a}_{x+k}^{a a} .
$$

When the additional medical inflation is $j_{k}^{[B]}=1 \%$ and the reserve is updated by $j_{k}^{[V]}=1 \%$ as in contract updating mechanism 2 , the actuarial equivalence is restored by increasing the premium by $j_{k}^{[P]}=1 \%$. This is the same premium update as for mechanism 1 as this mechanism sets the premium increase equal to the additional medical inflation, which we assume to be $1 \%$. By a similar reasoning the reserve increase resulting from updating mechanism 1 equals $j_{k}^{[V]}=1 \%$. We conclude that both updating mechanisms have the same impact on the reserve and premium in this setting.

\subsection{Reserve-dependent surrender values}

We illustrate the strategy proposed in Section 4.1 where the surrender values depend on the available reserve. The first column of Table 1 shows the initial premium for a contract with 

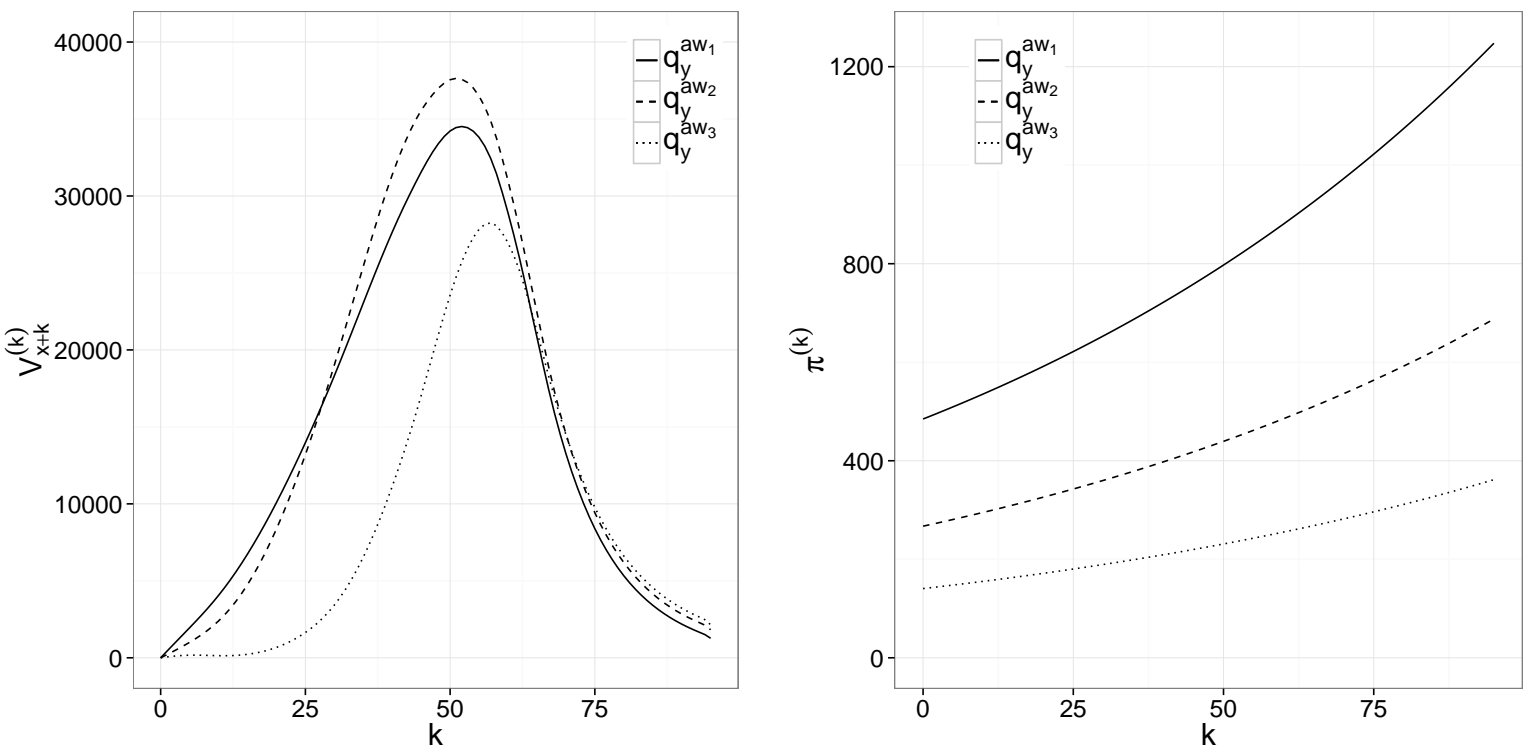

Figure 7: No surrender value: available reserves $V_{x+k}^{(k)}$ and premiums $\pi^{(k)}$ as calculated with the different types of lapse probabilities $q_{y}^{a w_{1}}$ (full line), $q_{y}^{a w_{2}}$ (dashed line), $q_{y}^{a w_{3}}$ (dotted line).

reserve-dependent surrender values under the different lapse probability assumptions. The initial premium of the contract decreases as the lapse probabilities increase. This is a consequence of the definition and choice of parameters $\beta_{k}$ and $\alpha_{k}$ used in the specification of the surrender values. As illustrated in Figure 8, the surrender values never exceed the available reserve. The part of the reserve not transferred in case of surrender can be added to the reserve of the remaining policyholders.
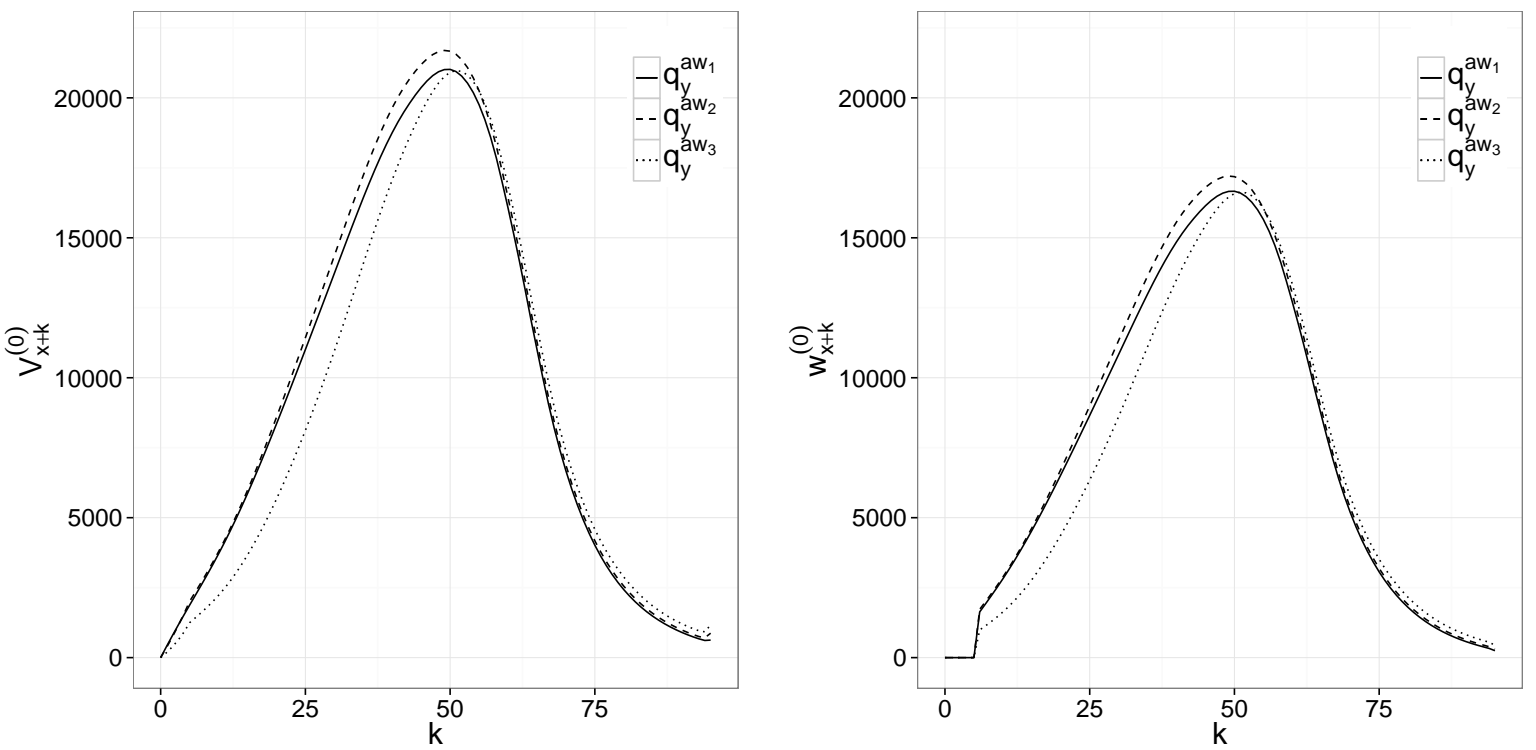

Figure 8: Reserve-dependent surrender value: available reserves $V_{x+k}^{(0)}(l e f t)$ and surrender values $w_{x+k}^{(0)}$ (right) as calculated with the different types of lapse probabilities $q_{y}^{a w_{1}}$ (full line), $q_{y}^{a w_{2}}$ (dashed line), $q_{y}^{a w_{3}}$ (dotted line). 

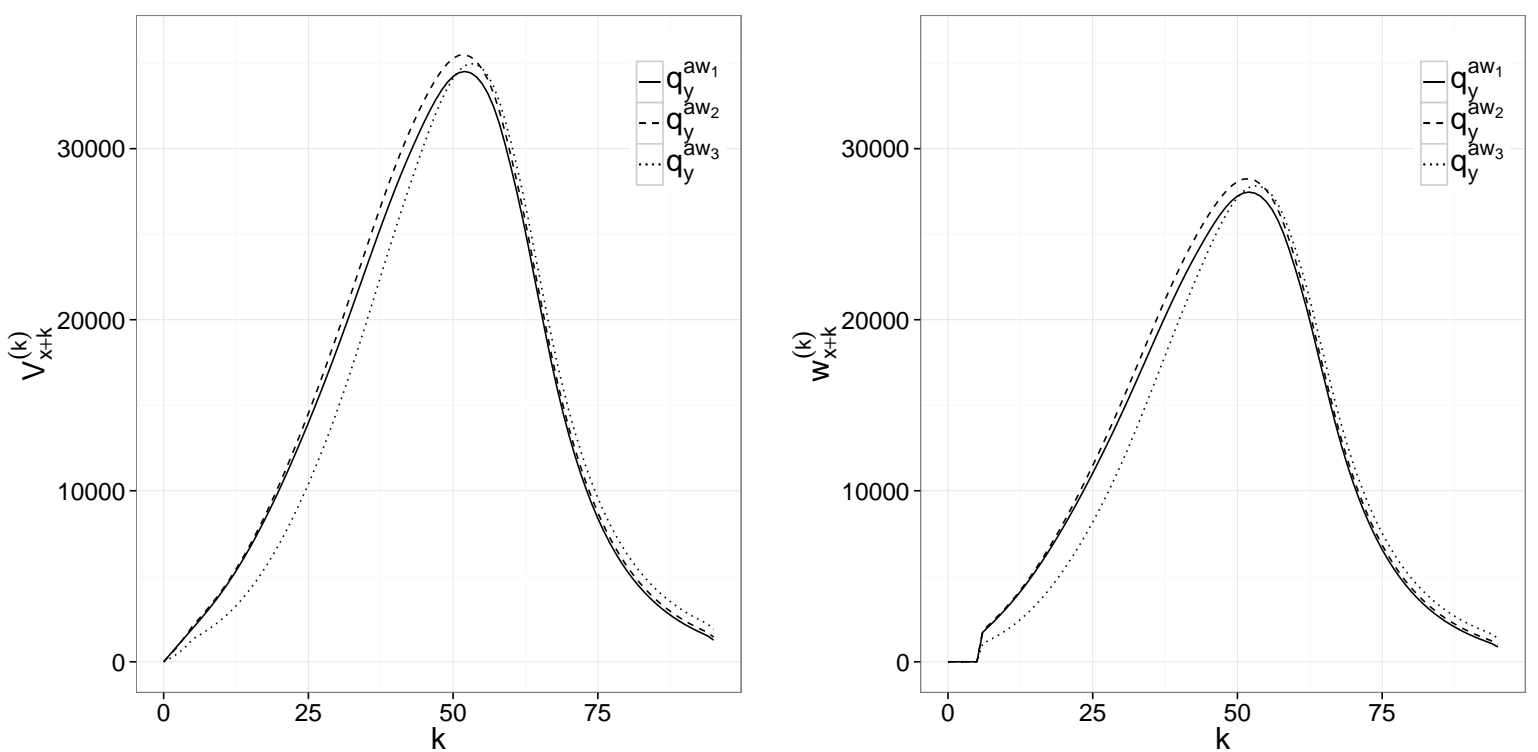

Figure 9: Reserve-dependent surrender value. Contract updating mechanism 1: available reserves $V_{x+k}^{(k)}$ (left) and surrender values $w_{x+k}^{(k)}($ right $)$ as calculated with the different types of lapse probabilities $q_{y}^{a w_{1}}$ (full line), $q_{y}^{a w_{2}}$ (dashed line), $q_{y}^{a w_{3}}$ (dotted line).
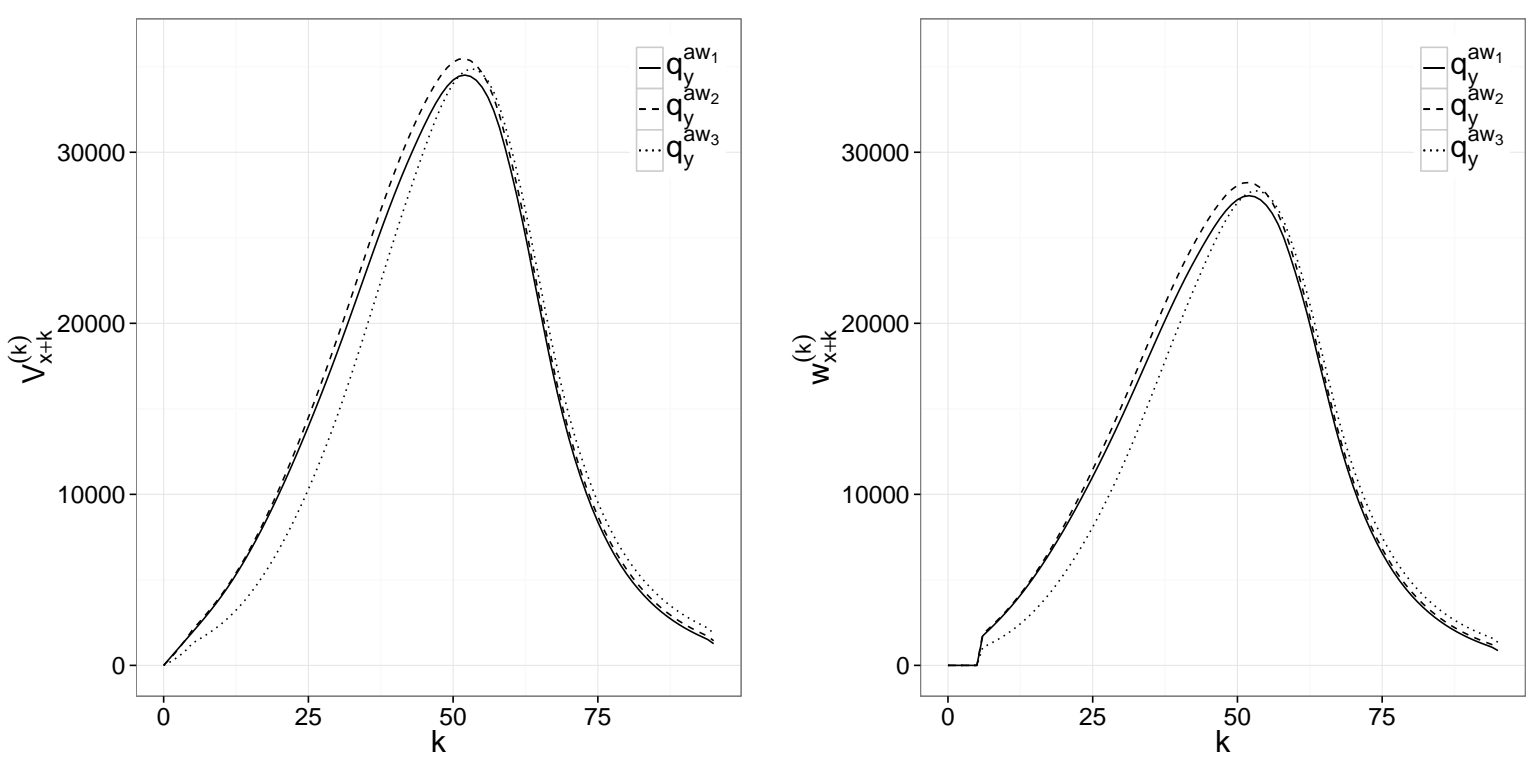

Figure 10: Reserve-dependent surrender value. Contract updating mechanism 2: available reserves $V_{x+k}^{(k)}($ left $)$ and surrender values $w_{x+k}^{(k)}($ right $)$ as calculated with the different types of lapse probabilities $q_{y}^{a w_{1}}$ (full line), $q_{y}^{a w_{2}}$ (dashed line), $q_{y}^{a w_{3}}$ (dotted line).

Figures 9 and 10 illustrate the evolution of the available reserve and surrender values over time under mechanisms 1 and 2, respectively. For both updating mechanisms the reserve increases over time due to the observed medical inflation. Therefore, the surrender values also increase because of their dependence on the reserve. The difference between both updating mechanisms is visualized in Figure 11. The graph on the right illustrates the yearly premium increase $j_{k}^{[P]}$ when 

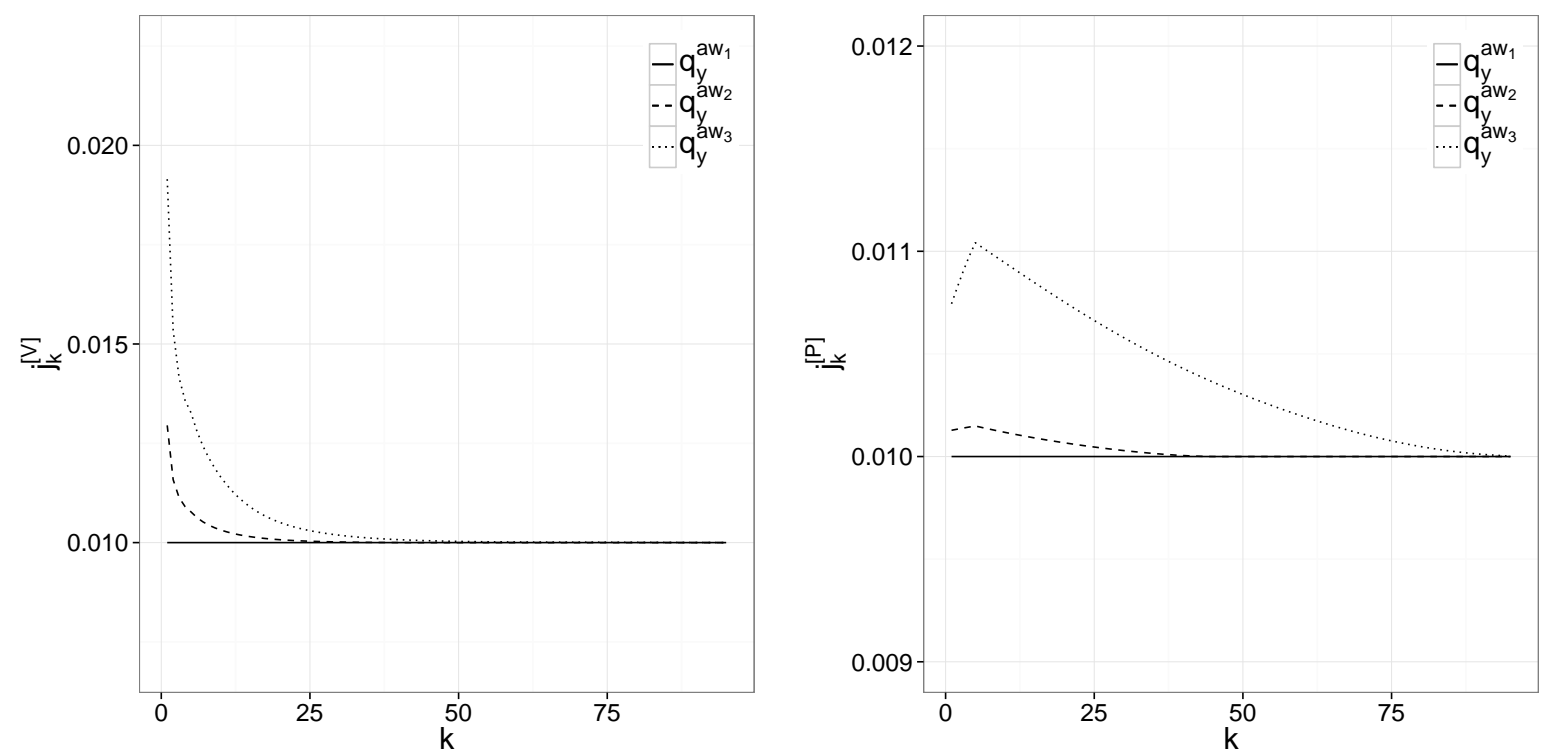

Figure 11: Reserve-dependent surrender value: yearly reserve increase $j_{k}^{[V]}$ for mechanism 1 (left) and yearly premium increase $j_{k}^{[P]}$ for updating mechanism 2 (right) as calculated with the different types of lapse probabilities $q_{y}^{a w_{1}}$ (full line), $q_{y}^{a w_{2}}$ (dashed line), $q_{y}^{a w_{3}}$ (dotted line).

we use mechanism 2 to account for observed medical inflation. The horizontal line at a premium increase of $1 \%(=0.01)$ corresponds to zero lapse probability $q_{y}^{a w_{1}}$. As discussed in Section 5.6, a zero lapse probability implies that the expected present value of the surrender values is zero at any time and the actuarial equivalence at time $k-1$ reduces to (60). For a reserve increase of $1 \%$ and the same increase in $B_{x+k}^{(k-1)}$ to account for observed medical inflation, the equivalence is restored by a premium increase of $1 \%$. The other lapse probabilities $\left(q_{y}^{a w_{2}}\right.$ and $\left.q_{y}^{a w_{3}}\right)$ decrease and converge to 0 over time. Consequently, over time the expected present value of the surrender values has a smaller impact on the premium when restoring the actuarial equivalence. Therefore the curves corresponding to lapse probabilities $q_{y}^{a w_{2}}$ and $q_{y}^{a w_{3}}$ also converge to $1 \%(=0.01)$ over time. The point at which the percentage starts to decrease corresponds to the year after which the surrender values are strictly positive.

The graph in the left panel of Figure 11 shows the increase of the reserve $j_{k}^{[V]}$ under mechanism 1. As for the right figure, the reduced equivalence relation (60) explains why the reserve increase for zero lapse probability $q_{y}^{a w_{1}}$ is constantly equal to $1 \%(=0.01)$ and why the reserve increases for the other lapse probabilities flatten out at 1\% $(=0.01)$.

\subsection{Premium-dependent surrender values}

We illustrate the strategy proposed in Section 4.2 where the surrender values directly depend on the premiums paid until the moment of surrender. The second column in Table 1 displays the initial premium $\pi^{(0)}$ calculated at policy issue with the different lapse probability assumptions in (47), (48) and (49). The evolution of the available reserve $V_{x+k}^{(0)}$ when medical inflation and contract updates over time are not taken into account is illustrated in the left graph of Figure 12. The right graph of this figure shows the evolution of surrender values (52) calculated at policy issue. If the assumptions in the technical basis at policy issue realize over time, this figure 
demonstrates that the surrender values never exceed the available reserve. As a consequence, higher lapse probabilities result in lower premiums as demonstrated in Table 1. A lower premium implies that the sum of the savings premiums is lower and gets negative sooner, so the cap of 0 in definition (51) of $\beta_{k}$ is reached more rapidly.
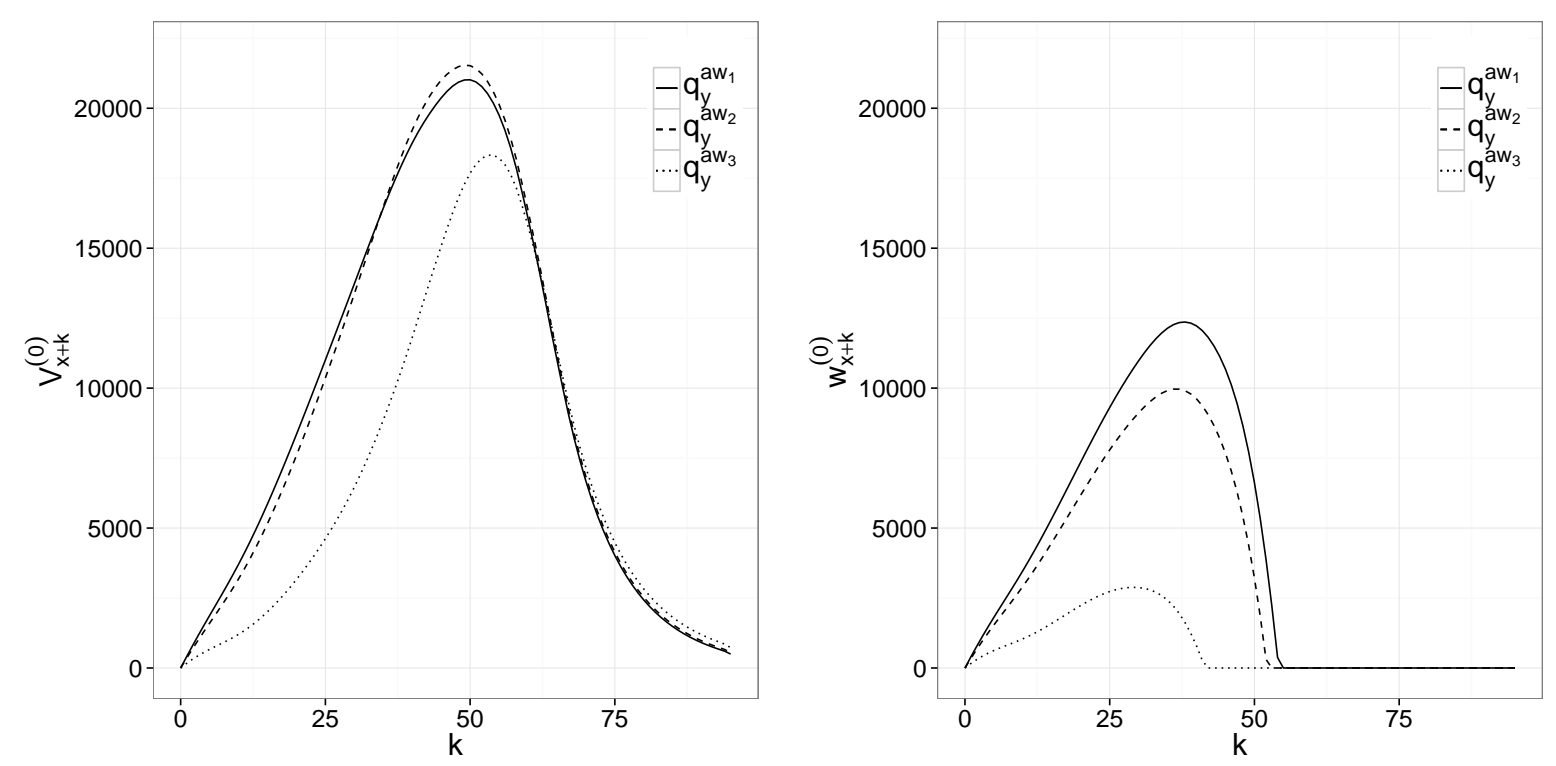

Figure 12: Premium-dependent surrender value: available reserves $V_{x+k}^{(0)}(l e f t)$ and surrender values $w_{x+k}^{(0)}$ (right) as calculated with the different types of lapse probabilities $q_{y}^{a w_{1}}$ (full line), $q_{y}^{a w_{2}}$ (dashed line), $q_{y}^{a w_{3}}$ (dotted line).

Figures 13 and 14 illustrate the impact of updating mechanisms 1 and 2, respectively, on the reserves and premium-based surrender values. As expected, for both mechanisms the reserves and surrender values have increased compared to their value computed at policy issue. However, surrender values never exceed the available reserve.

The difference between both updating mechanisms is highlighted in Figure 15. The right graph shows the yearly increase in the premium when we use updating mechanism 2 to account for observed medical inflation. When the lapse probability is zero the actuarial equivalence at time $(k-1)$ reduces to $(60)$. Additional medical inflation of $1 \%$ and a reserve update of $1 \%$ then require a premium increase of $1 \%(=0.01)$ to restore the actuarial equivalence. For the same reason the curves corresponding to the other lapse probabilities flatten out at $1 \%(=0.01)$. Indeed, due to the cap of zero on the $\beta_{k}$ the expected value of the surrender values drops to zero over time. Earlier on in the contract, we see that the premium increase is lower than the reserve increase. Using a similar reasoning, the reserve increase for $q_{y}^{a w_{1}}$ is constantly equal to $1 \%$ and the reserve increases for the other lapse probabilities flatten out at $1 \%(=0.01)$ in the left panel. Earlier on in the contract, the required reserve update for the non-zero lapse probabilities is lower than $1 \%$. 

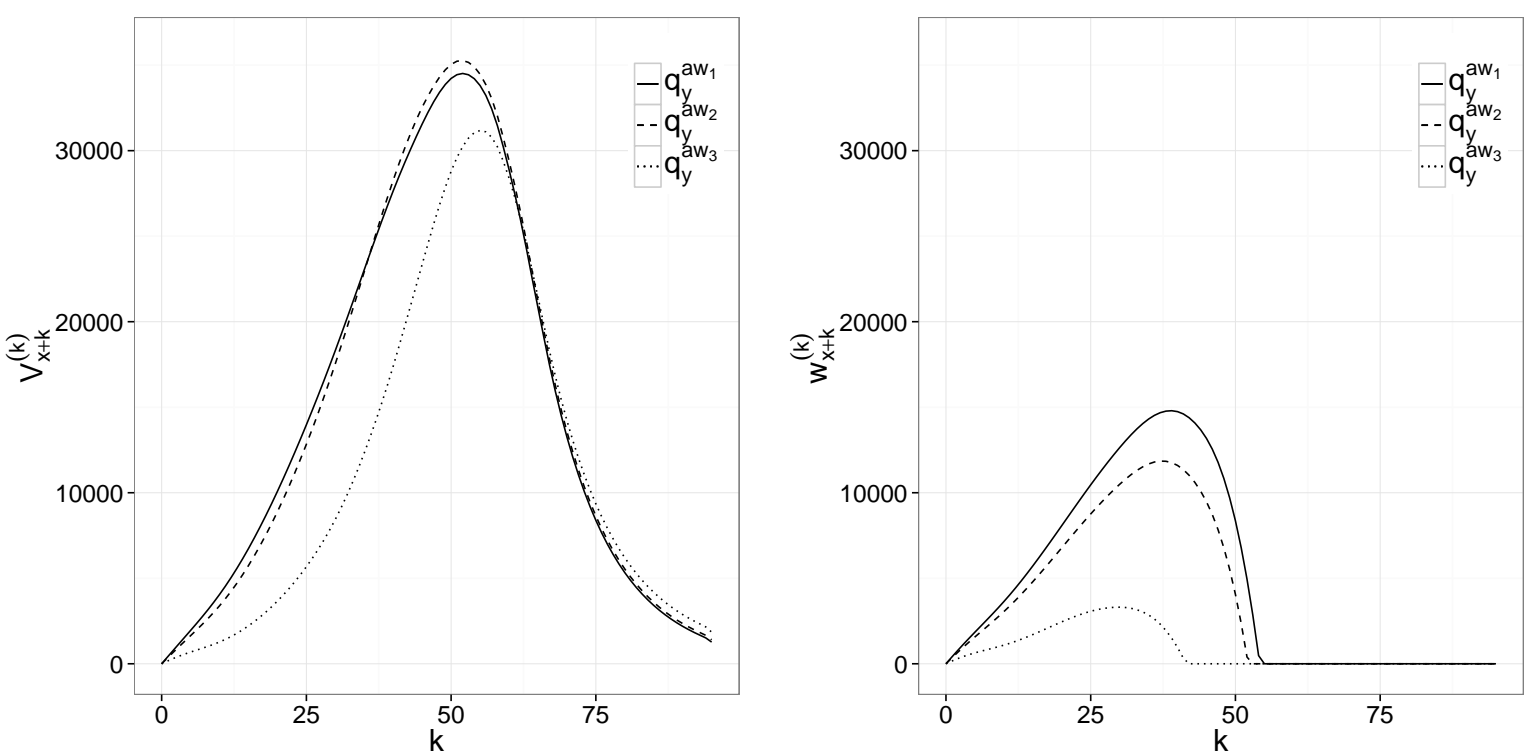

Figure 13: Premium-dependent surrender value. Updating mechanism 1: available reserves $V_{x+k}^{(k)}$ (left) and surrender values $w_{x+k}^{(k)}$ (right) as calculated with the different types of lapse probabilities $q_{y}^{a w_{1}}$ (full line), $q_{y}^{a w_{2}}$ (dashed line), $q_{y}^{a w_{3}}$ (dotted line).
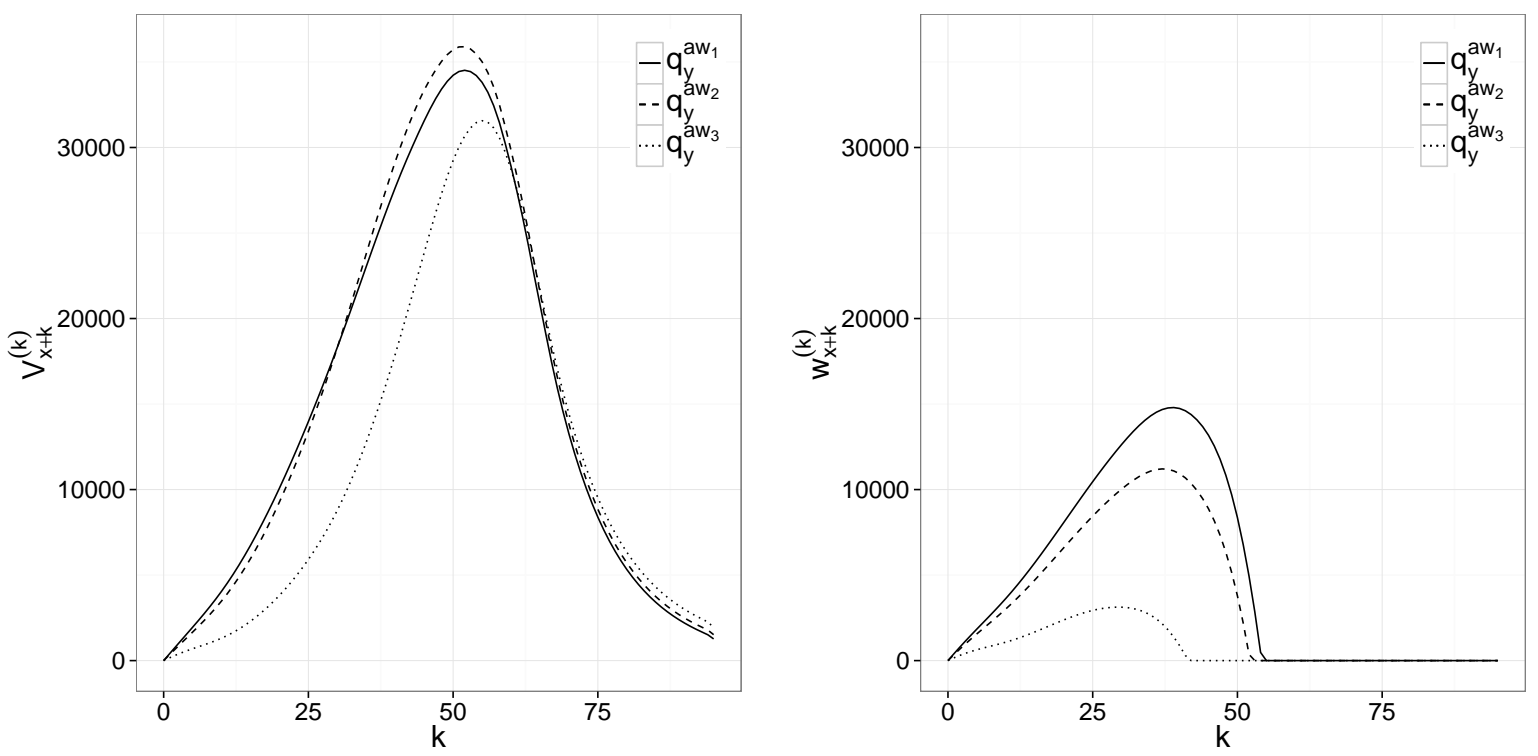

Figure 14: Premium-dependent surrender value. Updating mechanism 2: available reserves $V_{x+k}^{(k)}$ (left) and surrender values $w_{x+k}^{(k)}($ right $)$ as calculated with the different types of lapse probabilities $q_{y}^{a w_{1}}$ (full line), $q_{y}^{a w_{2}}$ (dashed line), $q_{y}^{a w_{3}}$ (dotted line).

\subsection{Discussion}

We put emphasis on the results of the sensitivity analysis regarding the lapse probabilities. Using information available at policy issue, Table 1 demonstrates that a higher lapse probability has a premium decreasing effect when the surrender benefit never exceeds the available reserve. 

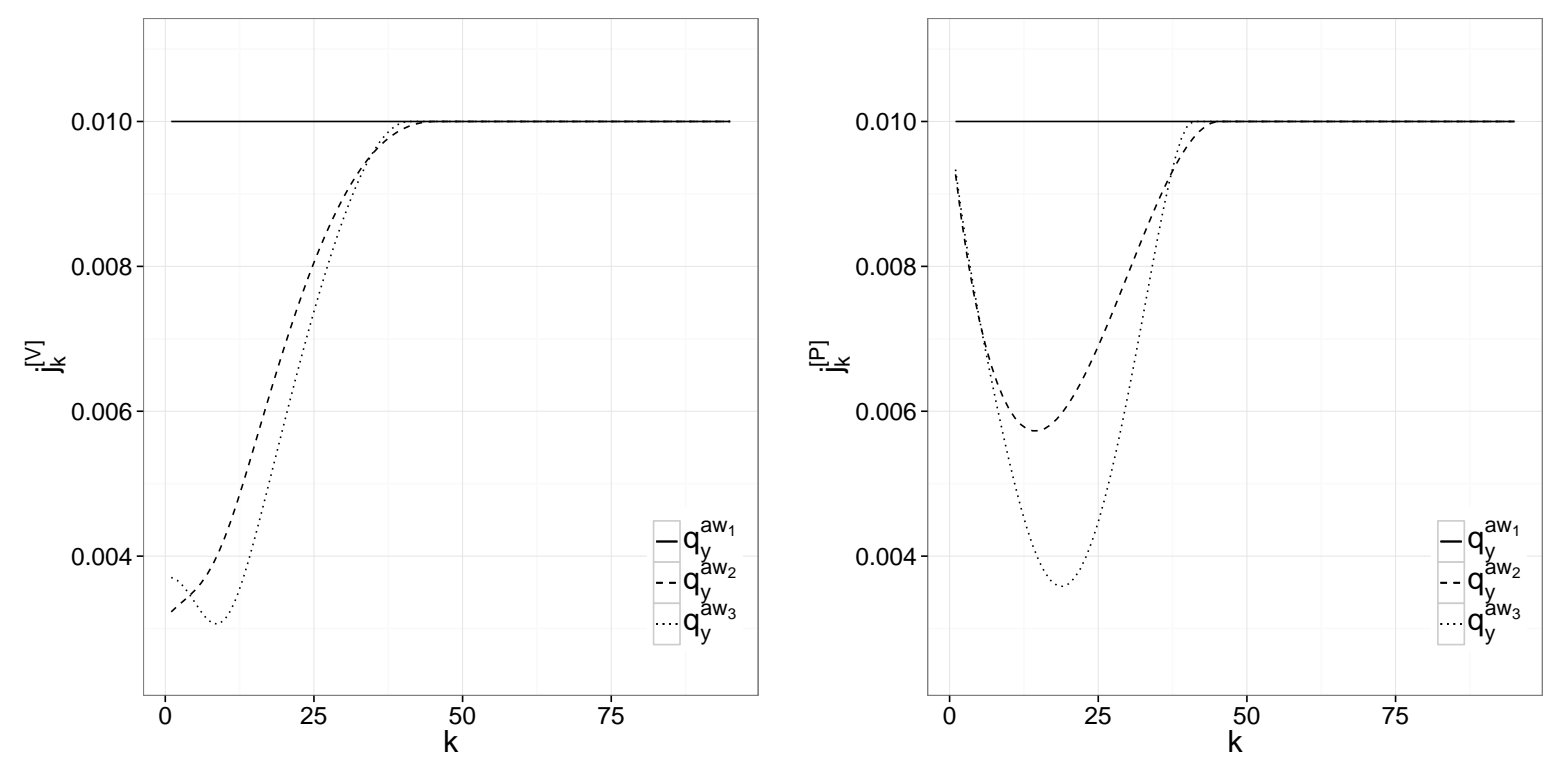

Figure 15: Premium-dependent surrender value: yearly reserve increase $j_{k}^{[V]}$ for mechanism 1 (left) and yearly premium increase $j_{k}^{[P]}$ for updating mechanism 2 (right) as calculated with the different types of lapse probabilities $q_{y}^{a w_{1}}$ (full line), $q_{y}^{a w_{2}}$ (dashed line), $q_{y}^{a w_{3}}$ (dotted line).

With increasing lapse rates the insurer expects a higher income from the part of the available reserve not paid out to the surrendering policyholder, and as a consequence the building up of the available reserve starts off slower. This is illustrated in Figures 6 and 7.

The impact of surrender probabilities is more complicated in Figures 8, 9, 10 and 12, 13, 14 as the reserve is indirectly influenced by the lapse probabilities not only through the initial premium, but also through the definition of the surrender values. Nevertheless, it is clear from these figures that the choice of lapse probabilities in the technical basis has a significant influence on the amount of the available reserve the insurer expects to hold over time.

Lastly, the consequences of the different updating mechanisms also clearly depend on the lapse probabilities as Figures 11 and 15 illustrate. The same updating mechanism can lead to very different relative increases under various lapse probability assumptions.

\section{Conclusion}

We consider a lifelong health insurance contract with level premiums. In contrast to Vercruysse et al. (2013) and Denuit et al. (2017) the policy under consideration allows for surrender values. The study of such contracts is relevant for two reasons: first, lifelong health insurance covers are present on the (European) market (with Belgium and Germany as prominent examples), and, second, the absence of a surrender value binds the insured to his insurer and creates a lock-in of policyholders, making it worthwhile to investigate actuarial models where surrender values are incorporated. We conclude that a careful design of the surrender value is imperative, and we present two meaningful specifications. In the first construction, the surrender value is a proportion of the available reserve minus a penalty. The advantage of this definition is that the surrender benefit will never exceed the available reserve. Disadvantages might be that not 
every insurer computes an individual reserve for each policyholder and that the concept of a reserve may seem obscure to a policyholder. In the second construction, the surrender value is a proportion of the premiums paid up to the moment of surrender (possibly with interest) minus a penalty. This definition is more tractable and understandable for the policyholder. For the insurer it is, however, not straightforward to guarantee that the surrender value will never exceed the available reserve on the contract. In our numerical examples we show how the premiumdependent surrender value can be designed in a way that satisfies this requirement. We study these lifelong health insurance covers with surrender values in a discrete-time framework where unanticipated medical inflation unfolds over time. Our contract allows for a yearly update of the premium and/or reserve, based on the observed inflation in the past year. We develop the conceptual framework of such updating mechanisms and provide explicit solutions for the initial premium and the updating strategy with the specific surrender values designed in this paper. In line with existing literature, we conclude that assumptions on lapse rates have a significant impact on the premium and reserve calculations, both at policy issue and throughout the yearly adjustments.

Our updating mechanism is easily extendable to other actuarial calculations in life and health insurance, where some elements of the technical basis are guaranteed while others are subject to revision according to policy conditions. Future research paths may focus on demonstrations of the updating mechanisms developed here to other life and health insurance products. The study of an appropriate index to capture medical inflation and its evolution over time, as well as research on data collection and statistical estimation of lapse rates, and the design of health insurance contracts allowing for surrender values, are other relevant topics for future work.

Acknowledgement. We thank four anonymous referees for their helpful comments, which substantially improved this article.

\section{References}

Assuralia (2016), 'Private ziekteverzekeraars - aantal verzekerde personen', http: //www.assuralia.be/fileadmin/content/stats/03_Cijfers_per_tak/05_Gezondheid/ 04_Aantal_verzekerden/NL/01_Aantal_verzekerden\%2001.htm.

Baumann, F., Meier, V. \& Werding, M. (2008), 'Transferable ageing provisions in individual health insurance contracts', German Economic Review 9, 287-311.

Christiansen, M., Denuit, M. \& Dhaene, J. (2014), 'Reserve-dependent benefits and costs in life and health insurance contracts', Insurance: Mathematics and Economics 57, 132-137.

Christiansen, M., Eling, M., Schmidt, J.-P. \& Zirkelbach, L. (2016), 'Who is changing health insurance coverage? Empirical evidence on policyholder dynamics', Journal of Risk and Insurance 83, 269-299.

Cochrane, J. (1995), 'Time-consistent health insurance', Journal of Political Economy 103, 445473.

Denuit, M., Dhaene, J., Hanbali, H., Lucas, N. \& Trufin, J. (2017), 'Updating mechanism for lifelong insurance contracts subject to medical inflation', European Actuarial Journal . 
Devolder, P., Denuit, M., Maréchal, X., Yerna, B.-L., Closon, J.-P., Léonard, C., Senn, A. \& Vinck, I. (2008), 'Setting a medical index for private health insurance contracts', KCE reports 96 . https://kce.fgov.be/publication/report/ setting-a-medical-index-for-private-health-insurance-contracts\#.VmLoY7_IYjU.

Dickson, D. C., Hardy, M. R. \& Waters, H. R. (2013), Actuarial mathematics for life contingent risks, Cambridge University Press.

Haberman, S. \& Pitacco, E. (1999), Actuarial models for disability insurance, CRC Press.

Hofmann, A. \& Browne, M. (2013), 'One-sided commitment in dynamic insurance contracts: Evidence from private health insurance in Germany', Journal of Risk and Uncertainty 46, 81112.

Kuo, W., Tsai, C. \& Chen, W.-K. (2003), 'An empirical study on the lapse rate: The cointegration approach', The Journal of Risk and Insurance 70, 489-508.

Milbrodt, H. (2005), Aktuarielle Methoden der deutschen Privaten Krankenversicherung, Verlag Versicherungswirtsch GmbH, Karlsruhe.

Pauly, M., Kunreuther, H. \& Hirth, R. (1995), 'Guaranteed renewability in insurance', Journal of Risk and Uncertainty 10, 143-156.

Pitacco, E. (2014), Health insurance: Basic actuarial models, Springer International Publishing.

Vercruysse, W., Dhaene, J., Denuit, M., Pitacco, E. \& Antonio, K. (2013), 'Premium indexing in lifelong health insurance', Far East Journal of Mathematical Sciences 2013, 365-384. 